\title{
Spectral rigidity of automorphic orbits in free groups
}

\author{
MATHiEu CARETte \\ STEFANO FRANCAVIGLIA \\ ILYA KAPOVICH \\ ARMANDO MARTINO
}

\begin{abstract}
It is well-known that a point $T \in \mathrm{cv}_{N}$ in the (unprojectivized) Culler-Vogtmann Outer space $\mathrm{cv}_{N}$ is uniquely determined by its translation length function $\|\cdot\|_{T}: F_{N} \rightarrow \mathbb{R}$. A subset $S$ of a free group $F_{N}$ is called spectrally rigid if, whenever $T, T^{\prime} \in \mathrm{cv}_{N}$ are such that $\|g\|_{T}=\|g\|_{T^{\prime}}$ for every $g \in S$ then $T=T^{\prime}$ in $\mathrm{cv}_{N}$. By contrast to the similar questions for the Teichmüller space, it is known that for $N \geq 2$ there does not exist a finite spectrally rigid subset of $F_{N}$.

In this paper we prove that for $N \geq 3$ if $H \leq \operatorname{Aut}\left(F_{N}\right)$ is a subgroup that projects to a nontrivial normal subgroup in $\operatorname{Out}\left(F_{N}\right)$ then the $H$-orbit of an arbitrary nontrivial element $g \in F_{N}$ is spectrally rigid. We also establish a similar statement for $F_{2}=$ $F(a, b)$, provided that $g \in F_{2}$ is not conjugate to a power of $[a, b]$.
\end{abstract}

20E08, 20F65; 57M07, 57M50, 53C24

\section{Introduction}

The phenomenon of marked length spectrum rigidity plays an important role in Riemannian geometry and adjacent areas. If $M$ is a closed manifold with a Riemannian metric $\rho$ of negative (but not necessarily constant) curvature, the associated marked length spectrum is the function $\ell_{\rho}: G \rightarrow \mathbb{R}$, where $G=\pi_{1}(M)$ and where for $\gamma \in G$ $\ell_{\rho}(\gamma)$ is the shortest length with respect to $\rho$ among all free homotopy representatives of $\gamma$. It is easy to see that $\ell_{\rho}(\gamma)=\ell_{\rho}\left(\gamma_{1}^{-1} \gamma \gamma_{1}\right)$ for all $\gamma, \gamma_{1} \in G$. Thus $\ell_{\rho}$ may be also viewed as a function from the set of conjugacy classes in $G$ to $\mathbb{R}$. One can also think of $\ell_{\rho}$ as the "translation length" function on $G$. Namely, let $X=\left(\widetilde{M}, d_{\rho}\right)$, where $d_{\rho}$ is the distance function on $\widetilde{M}$ corresponding to the lift $\widetilde{\rho}$ of $\rho$ to $\widetilde{M}$. Then the natural action of $G$ on $\widetilde{M}$ by covering transformation is an action of $G$ by isometries on $X$ (and thus can be thought of as a representation $G \rightarrow \operatorname{Isom}(X))$ and for every $\gamma \in G$ we have

$$
\ell_{\rho}(\gamma)=\inf _{x \in X} d_{\rho}(x, \gamma x)=\min _{x \in X} d_{\rho}(x, \gamma x)
$$

is the translation length of $\gamma$ as the isometry of $X$. 
The Marked Length Spectrum Rigidity Conjecture (MLSRC) states that knowing the function $\ell_{\rho}$ uniquely determines the isometry type of $(M, \rho)$. More precisely, the conjecture says that if $\rho, \rho^{\prime}$ are two smooth negatively curved Riemannian metrics on $M$ such that $\ell_{\rho}=\ell_{\rho^{\prime}}$ then there exists an isometry from $(M, \rho)$ to $\left(M, \rho^{\prime}\right)$ which is isotopic to the identity. This conjecture is known to hold in some special cases (more on this below), but is still open in full generality.

There are also various generalizations of MLSRC to other contexts, such as allowing more general types of metrics on $M$. There are also generalizations with the set-up where, given two representations $\tau: G \rightarrow \operatorname{Isom}\left(X_{1}\right)$ and $\tau_{2}: G \rightarrow \operatorname{Isom}\left(X_{2}\right)$ with the same marked length spectrum $\ell_{\tau_{1}}=\ell_{\tau_{2}}: G \rightarrow \mathbb{R}$ (where $X_{1}$ and $X_{2}$ are required to satisfy various kinds of negative or non-positive curvature conditions). Then the desired conclusion of MLSRC is that there exists an isometry $X_{1} \rightarrow X_{2}$ conjugating $\rho_{1}$ to $\rho_{2}$. MLSRC is known to hold for surfaces, including various generalizations of the types of metrics $\rho$ included under consideration (see Croke [17], Otal [51] and Croke, Fathi and Feldman [20]). There are also a number of known results establishing versions of MLSRC for representations $\rho_{1}: G \rightarrow \operatorname{Isom}\left(X_{1}\right)$ and $\rho_{2}: G \rightarrow \operatorname{Isom}\left(X_{2}\right)$ where $X_{1}, X_{2}$ are allowed to be higher dimensional, but there are more significant restrictions on the geometry of $X_{1}, X_{2}$ than in the results about MLSRC for surfaces (see, for example, Croke, Eberlein and Kleiner [19], Hersonsky and Paulin [34], Kim [47; 48; 49] and Dal'Bo and Kim [25]). However, the original version of MLSRC is still mostly open (except for rather special classes of metrics) in dimensions bigger than two. We refer the reader to the survey by Croke [18] for a more extended discussion on the topic.

In any context where MLSRC is known to hold, it is natural to ask if there are smaller subsets of $G$ such that knowing the restriction of the marked length spectrum to such a subset uniquely determines the entire marked length spectrum. Namely, for a given class of length functions $\ell: G \rightarrow \mathbb{R}$ where MLSRC is known to hold, say that a subset $S \subseteq G$ is spectrally rigid if whenever $\ell, \ell^{\prime}$ are two length functions from the class in question such that $\left.\ell\right|_{S}=\left.\ell^{\prime}\right|_{S}$ then $\ell=\ell^{\prime}$. For closed surfaces with metrics of constant curvature -1 the situation is particularly well-behaved. Thus it is known (see, for example, Fathi, Laudenbach and Poenaru [27]) that if $\Sigma$ is a closed oriented surface of genus $\geq 2$, then there exist elements $h_{1}, \ldots, h_{6 g-5} \in G=\pi_{1}(\Sigma)$ such that whenever $\rho_{1}, \rho_{2}$ are two points in the Teichmuller space $\mathcal{T}(\Sigma)$ (that is, marked hyperbolic metrics on $\Sigma$ ) such that $\ell_{\rho_{1}}\left(h_{i}\right)=\ell_{\rho_{2}}\left(h_{i}\right)$ for $i=1, \ldots, 6 g-5$ then $\ell_{\rho_{1}}=\ell_{\rho_{2}}$ and $\rho_{1}=\rho_{2}$ in $\mathcal{T}(\Sigma)$. Thus the subset $\left\{h_{1}, \ldots, h_{6 g-5}\right\} \subseteq G$ is "spectrally rigid" with respect to the class of hyperbolic metrics on $\Sigma$. We believe that looking for "small" spectrally rigid sets in other situations, where MLSRC is known to hold, is an interesting general problem worthy of further study. 
If $G$ is a finitely generated group acting by isometries on an $\mathbb{R}$-tree $X$, there is also a naturally associated translation length function $\|\cdot\|_{X}=\ell_{X}: G \rightarrow \mathbb{R}$, where for $g \in G$

$$
\ell_{X}(g)=\inf _{x \in X} d_{X}(x, g x)=\min _{x \in X} d_{X}(x, g x) .
$$

It is well-known (see Culler and Morgan [22], Paulin [52] and Chiswell [10]) that under some mild extra assumptions (which are satisfied, in particular, for the Outer space context discussed below), MLSRC holds, that is, knowing the function $\ell_{X}$ uniquely determines $X$ and the action of $G$ on $X$ (up to a $G$-equivariant isometry).

For a free group $F_{N}$ (where $N \geq 2$ ) the Culler-Vogtmann Outer space $\mathrm{cv}_{N}$ is an analog of the Teichmuller space of a hyperbolic surface. The space $\mathrm{cv}_{N}$ consists of minimal free discrete isometric actions of $F_{N}$ on $\mathbb{R}$-trees, considered up to $F_{N}-$ equivariant isometry. Every element $T \in \mathrm{cv}_{N}$ arises as the universal cover of a finite graph $\Gamma$, whose fundamental group is identified with $F_{N}$ via a particular isomorphism, where edges of $\Gamma$ are given positive real lengths and their lifts to $T$ are given the same lengths. There is an important subset, $\mathrm{CV}_{N} \subseteq \mathrm{cv}_{N}$, consisting of all $T \in \mathrm{cv}_{N}$ such that the quotient metric graph $T / F_{N}$ has volume 1 . The space $\mathrm{CV}_{N}$ is the projectivized Culler-Vogtmann Outer space, which was introduced by Culler and Vogtmann [23], before the introduction of $\mathrm{cv}_{N}$. Both $\mathrm{cv}_{N}$ and $\mathrm{CV}_{N}$ play an important role in the study of $\operatorname{Out}\left(F_{N}\right)$. We say that a subset $R \subseteq F_{N}$ is spectrally rigid if whenever $T_{1}, T_{2} \in \mathrm{cv}_{N}$ are such that $\|g\|_{T_{1}}=\|g\|_{T_{2}}$ for every $g \in R$ then $T_{1}=T_{2}$ in $\mathrm{cv}_{N}$. As noted above, $R=F_{N}$ is spectrally rigid. Moreover, for every $T \in \mathrm{cv}_{N}$ the translation length function $\|\cdot\|$ is constant on every conjugacy class in $F_{N}$. Thus if $R$ is chosen to consist of representatives of all conjugacy classes in $F_{N}$, then $R$ is spectrally rigid.

A surprising result of Smillie and Vogtmann [54] shows that there does not exist a finite spectrally rigid subset of $F_{N}$, where $N \geq 3$. A result of Cohen, Lustig and Steiner [13] establishes the same fact for $N=2$. In particular, it is proved in [54] that for any finite subset $R \subseteq F_{N}$, where $N \geq 3$, there exists a one-parametric family $\left(T_{t}\right)_{t \in[0,1]}$ of distinct points of $\mathrm{CV}_{N}$ such that for every $t \in[0,1]$ the length functions $\|\cdot\|_{T_{0}}$ and $\|\cdot\|_{T_{t}}$ agree on $R$. A similar statement follows from the recent work of Duchin, Leininger, and Rafi for flat metrics on surfaces of finite type [26]. Moreover, the paper [26] gives a complete characterization of when a set of simple closed curves on a finite type surface is spectrally rigid with respect to the space of flat metrics on that surface.

In view of the results of $[13 ; 54]$ it becomes interesting to look for infinite but "sparse" spectrally rigid subsets of $F_{N}$. The study of this topic was initiated by Kapovich in [38]. Namely, it is proved in [38] that if $A=\left\{a_{1}, \ldots, a_{N}\right\}$ is a free basis of $F_{N}$ then almost every trajectory of the simple non-backtracking random walk on $F_{N}$ with respect to 
$A$ yields a spectrally rigid subset of $F_{N}$. Recently Brian Ray [53] proved that for any $\phi \in \operatorname{Aut}\left(F_{N}\right)$ (where $N \geq 1$ ) and for any $g \in F_{N}$ the set $\langle\phi\rangle g=\left\{\phi^{n}(g): n \in \mathbb{Z}\right\}$ is not spectrally rigid in $F_{N}$. Ongoing work of Ray (unpublished) also shows that for any finite collection $H_{1}, \ldots, H_{m} \leq F_{N}$ of finitely generated subgroups of infinite index in $F_{N}$ (where $N \geq 2$ ) the set $H_{1} \cup \ldots \cup H_{m}$ is not spectrally rigid in $F_{N}$.

In the present paper we obtain a very different class of examples of spectrally rigid subsets of free groups from those constructed in [38]. We say that a subgroup $H \leq$ $\operatorname{Aut}\left(F_{N}\right)$ (where $N \geq 2$ ) is ample if the image of $H$ in $\operatorname{Out}\left(F_{N}\right)$ contains an infinite normal subgroup of $\operatorname{Out}\left(F_{N}\right)$. It is well-known (see Zimmermann [56] and Culler [21]) that every finite subgroup of $\operatorname{Out}\left(F_{N}\right)$ comes from a group of simplicial automorphisms of a finite connected graph without degree-one vertices and with fundamental group $F_{N}$. From here it is not hard to show that for $N \geq 3$ every nontrivial normal subgroup of $\operatorname{Out}\left(F_{N}\right)$ is infinite. For the case $N=2$ the group $\operatorname{Out}\left(F_{2}\right)$ possesses a unique nontrivial finite normal subgroup, namely the center $Z\left(\operatorname{Out}\left(F_{N}\right)\right)$ which is a cyclic group of order 2 generated by the "hyper-elliptic involution" of $F_{2}=F(a, b), a \mapsto a^{-1}$, $b \mapsto b^{-1}$.

Our main result is:

Theorem $\mathbf{A}$ Let $N \geq 2$ and let $H \leq \operatorname{Aut}\left(F_{N}\right)$ be an ample subgroup. Let $g \in F_{N}$ be an arbitrary nontrivial element; in the case $N=2$ we also assume that $g \in F_{2}=F(a, b)$ is not conjugate to a nonzero power of $[a, b]$ in $F_{2}$.

Then the orbit $H g=\{\phi(g): \phi \in H\}$ is a spectrally rigid subset of $F_{N}$.

Theorem A applies, for example, to the cases where $H=\operatorname{Aut}\left(F_{N}\right)$ (with $N \geq 2$ ) or where $H \leq \operatorname{Aut}\left(F_{N}\right)$ is the Torelli subgroup (with $N \geq 3$ ), that is, $H$ is the set of all elements of $\operatorname{Aut}\left(F_{N}\right)$ that act as the identity map on the abelianization $\mathbb{Z}^{N}$ of $F_{N}$. Theorem A also immediately implies that for $N \geq 3$ any $\operatorname{Aut}\left(F_{N}\right)$-invariant subset of $F_{N}$ with more than one element is spectrally rigid in $F_{N}$.

For $F_{2}=F(a, b)$ it is well-known that for every $\phi \in \operatorname{Aut}\left(F_{2}\right)$ the element $\phi([a, b])$ is conjugate to $[a, b]^{ \pm 1}$ in $F_{2}$, which easily implies that $\operatorname{Aut}\left(F_{2}\right)[a, b]$ is not spectrally rigid in $F_{2}$. However, as Theorem A shows, this example is essentially the only obstruction for spectral rigidity of an $\operatorname{Aut}\left(F_{N}\right)$-orbit of a nontrivial element in the case $N=2$.

As noted above, for any $T \in \mathrm{cv}_{N}\|\cdot\|_{T}$ is a class function and thus it can be viewed as a function on the set $\mathcal{C}_{N}$ of all the conjugacy classes of elements of $F_{N}$. The notion of a spectrally rigid set also naturally translates to subsets of $\mathcal{C}_{N}$. However, the results of Brian Ray about finitely generated subgroups of $F_{N}$ mentioned above, and the results 
of [38] about random walk trajectories are more naturally formulated in the setting of subsets of $F_{N}$. For that reason we adhere to that setting in this paper.

The first step in the proof of Theorem A is to establish Theorem 3.4 which says that the set $\mathcal{P}_{N}$ of all the primitive elements in $F_{N}$ is a spectrally rigid subset in $F_{N}$ for every $N \geq 2$. Recall that an element $g \in F_{N}$ is primitive if it belongs to some free basis of $F_{N}$. Thus $\mathcal{P}_{N}=\operatorname{Aut}\left(F_{N}\right) g$ where $g \in F_{N}$ is any primitive element. Theorem 3.4 is derived from the results of Francaviglia and Martino [29] about extremal Lipschitz distortions between two arbitrary points in $\mathrm{cv}_{N}$. A key fact there is that for any $T, T^{\prime} \in \mathrm{cv}_{N}$ the "extremal Lipschitz distortion" $D\left(T, T^{\prime}\right):=\sup _{g \in F_{N}, g \neq 1} \frac{\|g\|_{T^{\prime}}}{\|g\|_{T}}$ is actually a maximum which is realized by an element $g$ from some finite subset $\mathbf{U}_{T} \subseteq F_{N}$ depending only on $T$. Moreover, the explicit description of elements of $\mathbf{U}_{T}$ in [29] shows that they are all primitive, so that $\mathbf{U}_{T} \subseteq \mathcal{P}_{N}$. From here it is easy to see that if $\|g\|_{T}=\|g\|_{T^{\prime}}$ for every $g \in \mathcal{P}_{N}$ then $D\left(T, T^{\prime}\right)=D\left(T^{\prime}, T\right)=1$ and hence $T=T^{\prime}$ in $\mathrm{cv}_{N}$. A more careful version of the above argument yields the following "relative rigidity" result:

Theorem B Let $T \in \mathrm{cv}_{N}$ be arbitrary. There exists a finite set $S$ (depending on $T$ ) of primitive elements in $F_{N}$ with the following property: Whenever $T^{\prime} \in \mathrm{cv}_{N}$ is such that $\|g\|_{T^{\prime}}=\|g\|_{T}$ for every $g \in S$ then $T=T^{\prime}$ in $\mathrm{cv}_{N}$.

In fact, the proof of Theorem B shows that we can take $S=\mathbf{U}_{T}$.

After Theorem 3.4 is established, we derive Theorem A from Theorem 3.4 using the machinery of geodesic currents on free groups, and particularly exploiting the geometric intersection form between trees and currents, constructed by Kapovich [36] and by Kapovich and Lustig [40]. A geodesic current is a measure-theoretic analog of the notion of the conjugacy class in a (word-hyperbolic) group. Geodesic currents were introduced and studied by Bonahon [6;7] in the context of hyperbolic surfaces and the Teichmuller space, where they turned out to be quite useful. A geodesic current on a free group $F_{N}$ is a positive Radon measure $\mu$ on $\partial^{2} F_{N}=\partial F_{N} \times \partial F_{N}-$ diag which is "flip"-invariant and $F_{N}$-invariant. The space $\operatorname{Curr}\left(F_{N}\right)$ of all geodesic currents on $F_{N}$ comes equipped with a natural weak-* topology making it into a locally compact space, and with a natural left $\operatorname{Out}\left(F_{N}\right)$-action by linear transformations. The theory of geodesic currents on free groups has been actively developed in the last several years by Kapovich [35; 36; 37; 38] and Kapovich-Lustig [39; 40; 42; 41] (see also Bestvina and Feighn [3], Clay and Pettet [11], Hamenstädt [32], Francaviglia [28] and Kapovich and Nagnibeda $[44 ; 45 ; 46]$ for other recent applications of currents). The space $\operatorname{Curr}\left(F_{N}\right)$ turns out to be a natural counterpart for the Outer space $\mathrm{cv}_{N}$, and, more generally, the closure $\overline{\mathrm{cv}}_{N}$ of $\mathrm{cv}_{N}$. The closure $\overline{\mathrm{cv}}_{N}$ of $\mathrm{cv}_{N}$ (with respect to 
equivariant Gromov-Hausdorff convergence topology) is known to consist of all the minimal very small isometric actions of $F_{N}$ on $\mathbb{R}$-trees. The Outer space $\mathrm{cv}_{N}$ is an open $\operatorname{Out}\left(F_{N}\right)$-invariant dense subset of $\overline{\mathrm{cv}}_{N}$. It is again well-known that any point $T \in \overline{\mathrm{cv}}_{N}$ is uniquely determined by its translation length function $\|\cdot\|_{T}: F_{N} \rightarrow \mathbb{R}$.

The interaction between $\overline{\mathrm{cv}}_{N}$ and $\operatorname{Curr}\left(F_{N}\right)$ is given by the geometric intersection form

$$
\langle\cdot, \cdot\rangle: \overline{\mathrm{cv}}_{N} \times \operatorname{Curr}\left(F_{N}\right) \rightarrow \mathbb{R}_{\geq 0},
$$

constructed by Kapovich and Lustig [40]. The intersection form has a number of useful properties, such as being continuous, Out $\left(F_{N}\right)$-invariant, $\mathbb{R}_{\geq 0}$-linear with respect to the second argument and $\mathbb{R}_{\geq 0}$-homogeneous with respect to the first argument. Another key property of the intersection form, relating it to marked length spectra, is that for every $T \in \overline{\mathrm{cv}}_{N}$ and every $g \in F_{N}, g \neq 1$ we have

$$
\left\langle T, \eta_{g}\right\rangle=\|g\|_{T}
$$

where $\eta_{g} \in \operatorname{Curr}\left(F_{N}\right)$ is the so-called "counting" current defined by $g$. This last property is crucial in deriving Theorem A from Theorem 3.4.

As noted above, the first step in the proof of Theorem A is establishing Theorem 3.4, which is accomplished via analyzing extremal Lipschitz distortion between arbitrary points in $\mathrm{cv}_{N}$. After that the proof of Theorem A (for $N \geq 3$ ) proceeds as follows. It is known (see Kapovich and Lustig [39]) that for $N \geq 3$ there exists a unique minimal closed nonempty $\operatorname{Out}\left(F_{N}\right)$-invariant subset of the space $\mathbb{P} \operatorname{Curr}\left(F_{N}\right)$ of projectivized geodesic currents: this subset $\mathbb{M}_{N}$, called the minimal set, is exactly the closure in $\mathbb{P} \operatorname{Curr}\left(F_{N}\right)$ of the set of projective classes $\left[\eta_{a}\right]$ for all the primitive elements $a \in F_{N}$. Now let $H \leq \operatorname{Aut}\left(F_{N}\right)$ be an ample subgroup and let $g \in F_{N}$ be a nontrivial element. First, using a powerful recent result of Handel and Mosher [33] we conclude that $H$ contains a "fully irreducible" (also known as "iwip", which stands for "irreducible with irreducible powers") element $\psi \in H$. Then, using the assumption about $H$ being ample, and the dynamics of the action of fully irreducible automorphisms on $\mathbb{P} \operatorname{Curr}\left(F_{N}\right)$, we conclude that the closure in $\mathbb{P} \operatorname{Curr}\left(F_{N}\right)$ of the orbit $H\left[\eta_{g}\right]$ contains the minimal set $\mathbb{M}_{N}$. Then, using the intersection form $\langle\cdot, \cdot\rangle$ mentioned above, we conclude that for $T \in \mathrm{cv}_{N}$ knowing all the translation lengths $\|\phi(g)\|_{T}=\left\langle T, \eta_{\phi(g)}\right\rangle$, where $\phi \in H$, allows us to recover $\|a\|_{T}=\left\langle T, \eta_{a}\right\rangle$ for all primitive elements $a \in F_{N}$. Finally, by applying Theorem 3.4 we conclude that the set $\{\phi(g): \phi \in H\}$ is spectrally rigid in $F_{N}$.

The proof in Kapovich [38] that a random trajectory of the simple non-backtracking random walk on $F_{N}$ yields a spectrally rigid subset of $F_{N}$, was also based on using geodesic currents and the geometric intersection form. A key fact in that proof was that 
the $\mathbb{R}_{\geq 0}$-linear span of the set of counting currents $\eta_{w_{n}}$, where $\left(w_{n}\right)_{n \geq 1}$ is a random trajectory of the walk, is dense in $\operatorname{Curr}\left(F_{N}\right)$. A similar line of reasoning cannot be used to prove Theorem A. Indeed, if $A$ is a free basis of $F_{N}$ and $v$ is a freely reduced word over $A$, such that every freely reduced word of length 2 over $A$ occurs in $v$ as a subword, then $v$ cannot be a subword of a cyclic word representing a primitive element in $F_{N}$. This implies that $v$ has "weight 0" (see Kapovich [36] for the relevant terminology) in any finite linear combination of counting currents of primitive elements. Hence, by continuity, $v$ has "weight 0 " in every current from the closure $Z$ of the linear span of the counting currents of all primitive elements, and hence $Z$ is a proper subset of $\operatorname{Curr}\left(F_{N}\right)$.

In Section 6 we discuss a number of open problems motivated by the results of this paper, including questions about spectral rigidity properties of subgroups of $\operatorname{Out}\left(F_{N}\right)$, questions about spectral rigidity with respect to the closure $\overline{\mathrm{cv}}_{N}$ of $\mathrm{cv}_{N}$, and other problems.

Acknowledgments We are grateful to Martin Lustig for useful conversations and to Jing Tao for providing Example 6.2. We also thank the referee for useful suggestions. The first author is a Postdoctoral Researcher of the F.R.S.-FNRS (Belgium). The third author was supported by the NSF grant DMS-0904200.

\section{Preliminaries}

\subsection{Graphs and graph-related conventions}

Convention 2.1 (Graphs) A graph is a 1-complex. The set of 0-cells of a graph $\Delta$ is denoted $V \Delta$ and its elements are called vertices of $\Delta$. The closed 1-cells of a graph $\Delta$ are called topological edges of $\Delta$. The set of all topological edges is denoted $E_{\text {top }} \Delta$.

The interior of every topological edge is homeomorphic to the interval $(0,1) \subseteq \mathbb{R}$ and thus admits exactly 2 orientations (when considered as a 1-manifold). We call a topological edge endowed with the choice of an orientation on its interior an oriented edge of $\Delta$. The set of all oriented edges of $\Delta$ is denoted $E \Delta$. For an oriented edge $e \in E \Delta$ changing its orientation to the opposite produces another oriented edge of $\Delta$ denoted $e^{-1}$ and called the inverse of $e$. Thus ${ }^{-1}: E \Delta \rightarrow E \Delta$ is a fixed-point-free involution.

For every oriented edge $e$ of $\Delta$ there are naturally defined (and not necessarily distinct) vertices $o(e) \in V \Delta$, called the origin of $e$, and $t(e) \in E \Delta$, called the terminus of $e$, satisfying $o\left(e^{-1}\right)=t(e), t\left(e^{-1}\right)=o(e)$. 
An orientation on a graph $\Delta$ is a partition $E \Delta=E^{+} \Delta \sqcup E^{-} \Delta$, where for every $e \in E \Delta$ one of the edges $e, e^{-1}$ belongs to $E^{+} \Delta$ and the other edge belongs to $E^{-} \Delta$.

Definition 2.2 (Paths) A simplicial path or edge-path $\gamma$ of simplicial length $n \geq 1$ in $\Delta$ is a sequence of oriented edges

$$
\gamma=e_{1}, \ldots, e_{n}
$$

such that $t\left(e_{i}\right)=o\left(e_{i+1}\right)$ for $i=1, \ldots, n-1$. We say that $o(\gamma):=o\left(e_{1}\right)$ is the origin of $\gamma$ and that $t(\gamma)=t\left(e_{n}\right)$ is the terminus of $\gamma$. We also regard $\gamma=v \in V \Delta$ as an simplicial path in $\Gamma$ of simplicial length 0 with $o(\gamma)=t(\gamma)=v$. A simplicial path is called reduced if it does not contain a back-tracking, that is a subpath of the form $e e^{-1}$, where $e \in E \Delta$.

\subsection{Outer space}

The Culler-Vogtmann Outer space, introduced by Culler and Vogtmann in a seminal paper [23], is a free group analogue of the Teichmüller space of a closed surface of negative Euler characteristic. We briefly review some basics definitions and facts about the Outer space, and refer the reader to [23] and to Bestvina and Feighn [2], Bridson and Vogtmann [8], Guirardel [31] and Vogtmann [55] for more detailed background information on the topic.

Definition 2.3 (Non-projectivized Outer Space) Let $F_{N}$ be a finitely generated free group of rank $N \geq 2$.

The non-projectivized outer space $\mathrm{cv}_{N}$ consists of all minimal free and discrete isometric actions of $F_{N}$ on $\mathbb{R}$-trees. Two such trees $T_{1}, T_{2}$ are considered equal in $\mathrm{cv}_{N}$ if there exists an $F_{N}$-equivariant isometry between them. The space $\mathrm{cv}_{N}$ is endowed with the equivariant Gromov-Hausdorff convergence topology.

For $T \in \mathrm{cv}_{N}$ and $c>0$ denote by $c T \in \mathrm{cv}_{N}$ the tree that coincides with $T$ as a topological space and has the same $F$-action, but where the metric is multiplied by $c$.

A basic fact in the theory of Outer space states that every $T \in \mathrm{cv}_{N}$ is uniquely determined by its translation length function $\|\cdot\|_{T}: F_{N} \rightarrow \mathbb{R}$, where for every $g \in F_{N}$

$$
\|g\|_{T}=\min _{x \in T} d_{T}(x, g x)
$$

is the translation length of $g$. 
Proposition 2.4 Let $T_{1}, T_{2} \in \mathrm{cv}_{N}$ be such that $\|\cdot\|_{T_{1}}=\|\cdot\|_{T_{2}}$, that is $\|g\|_{T_{1}}=\|g\|_{T_{2}}$ for every $g \in F_{N}$. Then there exists an $F_{N}$-equivariant isometry between $T_{1}$ and $T_{2}$, so that $T_{1}=T_{2}$ in $\mathrm{cv}_{N}$.

Proposition 2.4 is a special case of a much more general fact. Thus it is known (see Paulin [52]) that for a finitely generated group $G$ any minimal irreducible (without a global fixed end) isometric action of $G$ on an $\mathbb{R}$-tree $T$ is uniquely determined (up to a $G$-equivariant isometry) by the translation length function $\|\cdot\|_{T}: G \rightarrow \mathbb{R}$ for this action.

Note that $\|g\|_{T}=\left\|h g h^{-1}\right\|_{T}$ for every $g, h \in F_{N}$. Thus $\|\cdot\|_{T}$ can be thought of as a function on the set of conjugacy classes in $F_{N}$. The space $\mathrm{cv}_{N}$ comes equipped with a natural right $\operatorname{Out}\left(F_{N}\right)$-action by homeomorphisms. At the length function level, if $\phi \in \operatorname{Out}\left(F_{N}\right), T \in \mathrm{cv}_{N}$ and $g \in F_{N}$ we have

$$
\|g\|_{T \phi}=\|\phi(g)\|_{T} .
$$

It is known that the equivariant Gromov-Hausdorff topology on $\mathrm{cv}_{N}$ coincides with the pointwise convergence topology at the level of length functions. Thus for $T_{n}, T \in \mathrm{cv}_{N}$ we have $\lim _{n \rightarrow \infty} T_{n}=T$ if and only if for every $g \in F$ we have $\lim _{n \rightarrow \infty}\|g\|_{T_{n}}=$ $\|g\|_{T}$.

Definition 2.5 (Projectivized Outer Space) Denote by $\mathrm{CV}_{N}$ the subset of $\mathrm{cv}_{N}$ consisting of all $T \in \mathrm{cv}_{N}$ such that the quotient graph $T / F_{N}$ has volume 1 .

The space $\mathrm{CV}_{N}$ is a closed $\operatorname{Out}\left(F_{N}\right)$-invariant subset of $\mathrm{cv}_{N}$ and it is called the projectivized Outer Space of $F_{N}$.

It is known that $\mathrm{CV}_{N}$ is $\operatorname{Out}\left(F_{N}\right)$-equivariantly homeomorphic to $\mathrm{cv}_{N} / \sim$, where $T_{1} \sim T_{2}$ if there is $c>0$ such that $T_{2}=c T_{1}$ in $\mathrm{cv}_{N}$. This fact justifies the term "projectivized Outer Space". For $T \in \mathrm{cv}_{N}$ we denote by $[T]$ the $\sim$-equivalence class of $T$ and call $[T]$ the projective class of $T$.

Points of $\mathrm{cv}_{N}$ have a more explicit combinatorial description as "marked metric graph structures" on $F$ :

Definition 2.6 (Metric graph) A metric graph is a graph $\Delta$ endowed with a metric structure $\mathcal{L}$, that is, a function $\mathcal{L}: E \Delta \rightarrow(0, \infty)$ such that for every $e \in E \Delta$ we have $\mathcal{L}(e)=\mathcal{L}\left(e^{-1}\right)$. The number $\mathcal{L}(e)$ is called the length of $e$ with respect to $\mathcal{L}$.

For a metric graph $(\Delta, \mathcal{L})$ its volume is defined as

$$
\operatorname{vol}_{\mathcal{L}}(\Delta)=\frac{1}{2} \sum_{e \in E \Delta} \mathcal{L}(e)=\sum_{e \in E^{+} \Delta} \mathcal{L}(e),
$$


where $E \Delta=E^{+} \Delta \sqcup E^{-} \Delta$ is any orientation on $\Delta$.

Definition 2.7 (Marking) Let $F_{N}$ be a free group of finite rank $N \geq 2$. A marking or a marked graph structure on $F_{N}$ is an isomorphism $\alpha: F_{N} \rightarrow \pi_{1}(\Gamma)$ where $\Gamma$ is a finite connected graph with the first Betti number equal to $N$ and such that $\Gamma$ has no degree- 1 and no degree- 2 vertices.

Definition 2.8 (Marked metric graph) A marked metric graph or a marked metric graph structure on $F_{N}$ consists of a marking $\alpha: F_{N} \rightarrow \pi_{1}(\Gamma)$ on $F_{N}$ together with a metric graph structure $\mathcal{L}$ on $\Gamma$.

Convention 2.9 Let $(\alpha, \mathcal{L})$ be a marked metric graph structure on $F_{N}$, where $\alpha: F_{N} \rightarrow$ $\pi_{1}(\Gamma, p)$ is a marking and where $\mathcal{L}$ is a metric structure on $\Gamma$.

Then $(\alpha, \mathcal{L})$ defines a point $T \in \operatorname{cv}_{N}$ as follows. Topologically, let $T=\widetilde{\Gamma}$, with an action of $F_{N}$ on $T$ via $\alpha$. We lift the metric structure $\mathcal{L}$ from $\Gamma$ to $T$ by giving every edge in $T$ the same length as that of its projection in $\Gamma$. This makes $T$ into an $\mathbb{R}$-tree equipped with a minimal free and discrete isometric action of $F_{N}$. (The assumption that $\Gamma$ has no degree-1 vertices guarantees that the action of $F_{N}$ on $T$ is minimal). Thus $T \in \mathrm{cv}_{N}$ and in this situation we will sometimes use the notation $T=(\alpha, \mathcal{L}) \in \mathrm{cv}_{N}$. Note that $T / F=\Gamma$.

Moreover, it is not hard to see that every point of $\mathrm{cv}_{N}$ arises in this fashion and that $\mathrm{CV}_{N}$ is exactly the set of all those $T=(\alpha, \mathcal{L}) \in \mathrm{cv}_{N}$ where $(\alpha, \mathcal{L})$ is a marked metric graph structure on $F_{N}$ with $\operatorname{vol}_{\mathcal{L}}(\Gamma)=1$.

For this reason we will also think of elements $T \in \mathrm{cv}_{N}$ as metric graphs, and the default assumption will be that every vertex of $T$ has degree $\geq 3$.

The following useful proposition is an immediate corollary of the definitions:

Proposition 2.10 Let $T \in \mathrm{cv}_{N}$ be realized by a marked metric graph structure $\left(\alpha: F_{N} \rightarrow \pi_{1}(\Gamma), \mathcal{L}\right)$ on $F_{N}$, so that $T=\left(\widetilde{\Gamma}, d_{\mathcal{L}}\right)$. Let $g \in F_{N}, g \neq 1$. Let $\gamma_{g}$ be the unique immersed circuit in $\Gamma$ obtained by reducing and cyclically reducing the edge-path $\alpha(g)$ in $\Gamma$.

Then $\|g\|_{T}$ is equal to the $\mathcal{L}$-length of $\gamma_{g}$.

The Outer space $\mathrm{cv}_{N}$ has a natural closure $\overline{\mathrm{cv}}_{N}$ with respect to the equivariant GromovHausdorff convergence topology (or, equivalently, with respect to the length function topology). It is known (see Bestvina and Feighn [2], Cohen and Lustig [12] and Guirardel [30]) that $\overline{\mathrm{cv}}_{N}$ consists precisely of (the $F_{N}$-equivariant isometry classes of) 
all the very small minimal isometric actions of $F_{N}$ on $\mathbb{R}$-trees. The $\operatorname{Out}\left(F_{N}\right)$-action on $\mathrm{cv}_{N}$ naturally extends to an action on $\overline{\mathrm{cv}}_{N}$ by homeomorphisms. Moreover, the projectivization $\overline{\mathrm{CV}}_{N}$ of $\overline{\mathrm{cv}}_{N}$ is compact and contains (a copy of) $\mathrm{CV}_{N}$ as an open dense $\operatorname{Out}\left(F_{N}\right)$-invariant subset. The space $\overline{\mathrm{CV}}_{N}$ is sometimes called the Thurston compactification of $\mathrm{CV}_{N}$.

\section{Extremal Lipschitz distortions and rigidity of the set of primitive elements}

For $N \geq 2$ we denote by $\mathcal{P}_{N}$ the set of all primitive elements in $F_{N}$.

Notation 3.1 Let $T \in \mathrm{cv}_{N}$ and $T^{\prime} \in \overline{\mathrm{cv}}_{N}$. Denote

$$
D\left(T, T^{\prime}\right):=\sup _{g \in F_{N}, g \neq 1} \frac{\|g\|_{T^{\prime}}}{\|g\|_{T}} .
$$

Notation 3.2 (Almost simple curves) Let $T \in \mathrm{cv}_{N}$ and let $\Gamma=T / F_{N}$ be the quotient metric graph, with the metric structure $\mathcal{L}$ coming from $T$. Thus $F_{N}$ is naturally identified with $\pi_{1}(\Gamma)$ via an isomorphism $\alpha: F_{N} \rightarrow \pi_{1}(\Gamma)$ and $T=(\alpha, \mathcal{L})$ in $\mathrm{cv}_{N}$.

Let $\mathbf{U}_{T} \subseteq F_{N}$ be the set of all elements of $F_{N}$ corresponding (under $\alpha$ ) to the closed curves $\gamma$ in $\Gamma$ of one of the following types:

(1) $\gamma$ is a nontrivial simple closed circuit in $\Gamma$;

(2) $\gamma=\gamma_{1} \gamma_{2}$ is a concatenation of two nontrivial simple closed circuits $\gamma_{1}, \gamma_{2}$, each beginning and ending at a common vertex $v$, and such that $\gamma_{1}, \gamma_{2}$ do not contain any common topological edges. We refer to such $\gamma$ as figure-eight curves in $T / F_{N}$.

(3) $\gamma$ is a "barbell" circuit, that is $\gamma=\gamma_{1} \beta \gamma_{2} \beta^{-1}$ where $\gamma_{i}$ is a nontrivial simple closed circuit at a vertex $v_{i}$ of $\Gamma$ with $v_{1} \neq v_{2}$, where $\beta$ is a simple edge-path from $v_{1}$ to $v_{2}$ in $\Gamma$ and where $\gamma_{1}, \beta, \beta_{2}$ do not have any common topological edges. We refer to such $\gamma$ as barbell curves in $T / F_{N}$.

Note that, by construction, every element of $\mathbf{U}_{T}$ is primitive in $F_{N}$ and the set $\mathbf{U}_{T}$ is finite. Moreover $\# \mathbf{U}_{T} \leq K(N)$ for some constant $K(N)$ depending only on $N$. We call elements of $\mathbf{U}_{T}$ almost simple curves for $T / F_{N}$.

We need the following fact established by Francaviglia and Martino in [29]: 
Proposition 3.3 Let $T, T^{\prime} \in \mathrm{cv}_{N}$ be arbitrary. Then

$$
D\left(T, T^{\prime}\right)=\max _{g \in \mathbf{U}_{T}} \frac{\|g\|_{T^{\prime}}}{\|g\|_{T}} .
$$

Thus we see that the supremum in the definition of $D\left(T, T^{\prime}\right)$ in $(t)$ is a maximum and it is achieved on one of elements from the finite subset $\mathbf{U}_{T} \subseteq \mathcal{P}_{N}$.

Proposition 3.3 quickly implies that the set of all primitive elements is spectrally rigid:

Theorem 3.4 Let $N \geq 2$. Then the set $\mathcal{P}$ of all primitive elements in $F_{N}$ is spectrally rigid in $F_{N}$.

Proof Let $T, T^{\prime} \in \mathrm{cv}_{N}$ be such that $\|g\|_{T}=\|g\|_{T^{\prime}}$ for every $g \in \mathcal{P}_{N}$.

Then

$$
D\left(T, T^{\prime}\right)=\sup _{g \in F_{N}, g \neq 1} \frac{\|g\|_{T^{\prime}}}{\|g\|_{T}}=\max _{g \in \mathbf{U}_{T}} \frac{\|g\|_{T^{\prime}}}{\|g\|_{T}}=1
$$

since $\mathbf{U}_{T} \subseteq \mathcal{P}_{N}$. Similarly,

$$
D\left(T^{\prime}, T\right)=\sup _{g \in F_{N}, g \neq 1} \frac{\|g\|_{T}}{\|g\|_{T^{\prime}}}=\max _{g \in \mathbf{U}_{T^{\prime}}} \frac{\|g\|_{T}}{\|g\|_{T^{\prime}}}=1
$$

so that

$$
\inf _{g \in F_{N}, g \neq 1} \frac{\|g\|_{T^{\prime}}}{\|g\|_{T}}=\sup _{g \in F_{N}, g \neq 1} \frac{\|g\|_{T^{\prime}}}{\|g\|_{T}}=1 .
$$

Thus $\|g\|_{T}=\|g\|_{T^{\prime}}$ for every $g \in F_{N}$ and hence $T=T^{\prime}$ in $\mathrm{cv}_{N}$, as required.

We now state a more precise (compared to Proposition 3.3) statement summarizing some of the results of Francaviglia and Martino [29]:

Proposition 3.5 Let $T, T^{\prime} \in \mathrm{cv}_{N}$ be arbitrary. Let $L:=D\left(T, T^{\prime}\right)$. Then there exists an $F_{N}$-equivariant $L$-Lipschitz map $f: T \rightarrow T^{\prime}$ with the following properties:

(1) On each edge $e$ of $T$ the map $f$ is a linear map with constant stretch $D_{e} \geq 0$ (note that, because of $F_{N}$-equivariance, $D_{e_{1}}=D_{e_{2}}$ whenever $e_{1}$ and $e_{2}$ are in the same $F_{N}$-orbit of edges of $T$ ).

(2) There exists $h \in \mathbf{U}_{T}$ such that for every edge $e$ in the axis $A_{h}$ of $h$ in $T$ we have $D_{e}=L$ and the restriction of $f$ to $A_{h}$ is injective (so that $\left.f\right|_{A_{h}}$ is an $L$-homothety).

We can now establish Theorem B from the Introduction: 
Theorem 3.6 Let $T, T^{\prime} \in \mathrm{cv}_{N}$ be such that $\|g\|_{T}=\|g\|_{T^{\prime}}$ for every $g \in \mathbf{U}_{T}$. Then $T^{\prime}=T$ in $\mathrm{cv}_{N}$.

Proof Let $T, T^{\prime} \in \mathrm{cv}_{N}$ be such that $\|g\|_{T}=\|g\|_{T^{\prime}}$ for every $g \in \mathbf{U}_{T}$. Hence by Proposition 3.3 $D\left(T, T^{\prime}\right)=1$. Let $f: T \rightarrow T^{\prime}$ be an $F_{N}$-equivariant 1-Lipschitz map provided by Proposition 3.5. In particular, $D_{e} \leq 1$ for every edge $e$ of $T$.

We claim that $D_{e}=1$ for every edge $e$ of $T$. Indeed, suppose not, so that there exists an edge $e_{0}$ of $T$ with $D_{e_{0}}<1$. Let $y_{0}$ be the edge of $T / F_{N}$ which is the projection of $e_{0}$ to $T / F_{N}$. Then there exists an immersed circuit $\gamma_{0}$ in $T / F_{N}$ passing through the edge $y_{0}$ such that $\gamma_{0}$ is an almost simple curve in $F_{N} / T$. Let $g_{0} \in F_{N}$ correspond to $\gamma_{0}$, so that $g_{0} \in \mathbf{U}_{T}$. The fact that $D_{e_{0}}<1$ and that $D_{e} \leq 1$ for every edge $e$ of $T$ implies that $\left\|g_{0}\right\|_{T^{\prime}}<\left\|g_{0}\right\|_{T}$. Again, this contradicts our assumptions on $T, T^{\prime}$ and the fact that $g_{0} \in \mathbf{U}_{T}$.

We now claim that $f$ is injective. Indeed, suppose not. Then there exist two distinct oriented edges $e_{1}, e_{2}$ of $T$ with a common initial vertex $v$ such that $f$ "folds" a non-degenerate initial segment of $e_{1}$ and a non-degenerate initial segment of $e_{2}$. Let $y_{1}, y_{2}$ be the edges of $T / F_{N}$ which are the projections of $e_{1}$ and $e_{2}$ respectively to $T / F_{N}$. From the definition of an almost simple curve for $T / F_{N}$ it follows that there exists an immersed circuit $\gamma$ in $T / F_{N}$ containing $y_{1}^{-1} y_{2}$ as a subpath such that $\gamma$ is an almost simple curve in $T / F_{N}$. Thus $\gamma$ corresponds to $g \in \mathbf{U}_{T}$. By $F_{N}$-equivariance of $f$ we may assume that $e_{1}^{-1} e_{2}$ is a subpath of the axis of $g$ in $T$. Since $D_{e}=1$ for every edge $e$ of $T$ and since $f$ folds nondegenerate initial segments of $e_{1}$ and $e_{2}$, it follows that $\|g\|_{T^{\prime}}<\|g\|_{T}$. This contradicts the fact that $g \in \mathbf{U}_{T}$ and that by assumption $\|g\|_{T}=\|g\|_{T^{\prime}}$. Hence $f$ is injective as claimed.

Thus the map $f$ is injective and is isometric on every edge of $T$. Therefore $f: T \rightarrow T^{\prime}$ is an isometric embedding. Since the actions of $F_{N}$ on $T$ and $T^{\prime}$ are minimal, it follows that $f(T)=T^{\prime}$, so that $f: T \rightarrow T^{\prime}$ is an $F_{N}$-equivariant isometry. Hence $T=T^{\prime}$ in $\mathrm{cv}_{N}$, as required.

\section{Geodesic currents}

\subsection{Basic facts}

We will only state a few basic facts and definitions about currents, and refer the reader to Martin [50], Kapovich [35; 36; 37] and Kapovich and Lustig [39; 40; 42] for more detailed background information regarding geodesic currents. 
For the free group $F_{N}$ define its "double boundary" $\partial^{2} F_{N}$ as

$$
\partial^{2} F_{N}:=\partial F_{N} \times \partial F_{N}-\operatorname{diag}=\left\{(\xi, \zeta) \in \partial F_{N} \times \partial F_{N}: \xi \neq \zeta\right\} .
$$

The space $\partial^{2} F_{N}$ comes equipped with a natural topology, inherited from $\partial F_{N} \times \partial F_{N}$, and a natural translation action of $F_{N}$ by homeomorphisms. There is also a natural "flip" map $\partial^{2} F_{N} \rightarrow \partial^{2} F_{N},(\xi, \zeta) \mapsto(\zeta, \xi)$, interchanging the two coordinates on $\partial^{2} F_{N}$.

Recall that a geodesic current on $F_{N}$ is a positive Radon measure $\mu$ on $\partial^{2} F_{N}$ which is $F_{N}$-invariant and flip-invariant. Here the "flip" map $\partial^{2} F_{N} \rightarrow \partial^{2} F_{N},(X, Y) \mapsto(Y, X)$ interchanges the two coordinates of $\partial^{2} F_{N}$. The space $\operatorname{Curr}\left(F_{N}\right)$ of all geodesic currents on $F_{N}$ comes equipped with a natural weak-* topology and a natural left $\operatorname{Aut}\left(F_{N}\right)$-action by $\mathbb{R}_{\geq 0}$-linear homeomorphisms. The group of inner automorphisms of $F_{N}$ is contained in the kernel of this action, and hence the $\operatorname{Aut}\left(F_{N}\right)$ action naturally factors through to the action of $\operatorname{Out}\left(F_{N}\right)$ on $\operatorname{Curr}\left(F_{N}\right)$.

Every nontrivial element $g \in F_{N}$ defines a counting current $\eta_{g} \in \operatorname{Curr}\left(F_{N}\right)$, which turns out to depend only on the conjugacy class $[g]$ of $g$ in $F_{N}$. Although the explicit definition of $\eta_{g}$ is not directly relevant for this paper, we briefly recall one of the equivalent definitions of $\eta_{g}$ here. Suppose first that $g \in F_{N}$ is a nontrivial element which is not a proper power in $F_{N}$. There are two well-defined distinct "poles" $g^{\infty}, g^{-\infty} \in \partial F_{N}$ where

$$
g^{\infty}=\lim _{n \rightarrow \infty} g^{n}, \quad g^{-\infty}=\lim _{n \rightarrow \infty} g^{-n}
$$

where the convergence is understood in the sense of the standard hyperbolic compactification $F_{N} \cup \partial F_{N}$ of $F_{N}$. Then $\left(g^{-\infty}, g^{\infty}\right) \in \partial^{2} F_{N}$. Let $[g]$ denote the conjugacy class of $g$ in $F_{N}$. Then

$$
\eta_{g}:=\sum_{h \in[g] \cup\left[g^{-1}\right]} \delta_{\left(h^{-\infty}, h^{\infty}\right)} .
$$

Now if $g \in F_{N}$ is an arbitrary nontrivial element, $g$ can be uniquely written as $g=g_{0}^{m}$ where $m \geq 1$ and $g_{0} \in F_{N}$ is not a proper power. Then $\eta_{g}$ is defined as $\eta_{g}:=m \eta_{g_{0}}$. We summarize the following basic facts about counting currents (see Kapovich [36]):

Proposition 4.1 Let $N \geq 2$ and let $g \in F_{N}, g \neq 1$. Then

(1) We have $\eta_{g}=\eta_{g^{-1}}$.

(2) For every $n \in \mathbb{Z}, n \neq 0$, we have $\eta_{g^{n}}=|n| \eta_{g}$.

(3) For every $h \in[g]$ we have $\eta_{g}=\eta_{h}$. Thus $\eta_{g}$ depends only on the conjugacy class of $g$, so we also use the notation $\eta_{[g]}:=\eta_{g}$. 
(4) For every $\phi \in \operatorname{Out}\left(F_{N}\right)$ we have $\phi \eta_{[g]}=\eta_{\phi([g])}$.

The scalar multiples of counting currents are called rational currents. An important basic fact states that the set $\mathcal{R}_{N}:=\left\{r \eta_{g} \mid r \geq 0, g \neq 1, g \in F_{N}\right\}$ of all rational currents is dense in $\operatorname{Curr}\left(F_{N}\right)$. The space $\operatorname{Curr}\left(F_{N}\right)$ has a natural projectivization $\mathbb{P} \operatorname{Curr}\left(F_{N}\right)$ consisting of all equivalence classes $[\mu]$ where $\mu \in \operatorname{Curr}\left(F_{N}\right), \mu \neq 0$. Here two currents $\mu_{1}, \mu_{2}$ are equivalent if there exists $r>0$ such that $\mu_{2}=r \mu_{1}$. The equivalence class $[\mu]$ is also called the projective class of $\mu$. The space $\mathbb{P} \operatorname{Curr}\left(F_{N}\right)$ is compact and it inherits a left action of $\operatorname{Out}\left(F_{N}\right)$ by homeomorphisms. A basic fact about geodesic currents states (see Kapovich [36]):

Proposition 4.2 Let $N \geq 2$ and let $g, h \in F_{N}$ be nontrivial elements. Then $\left[\eta_{g}\right]=\left[\eta_{h}\right]$ in $\mathbb{P} \operatorname{Curr}\left(F_{N}\right)$ if and only if there exist $u \in F_{N}, m, n \in \mathbb{Z}$ such that $[g]=\left[u^{m}\right]$ and $[h]=\left[u^{n}\right]$.

\subsection{The geometric intersection form}

A key object connecting the Outer space and the space of geodesic currents is the so-called geometric intersection form, constructed by Kapovich and Lustig [40]:

Proposition 4.3 Let $N \geq 2$. There exists a unique continuous map

$$
\langle\cdot, \cdot\rangle: \overline{\operatorname{cv}}_{N} \times \operatorname{Curr}\left(F_{N}\right) \rightarrow \mathbb{R}_{\geq 0}
$$

with the following properties:

(1) $\left\langle T, c_{1} \mu_{1}+c_{2} \mu_{2}\right\rangle=c_{1}\left\langle T, \mu_{1}\right\rangle+c_{2}\left\langle T, \mu_{2}\right\rangle$ for any $T \in \overline{\mathrm{cv}}_{N}, \mu_{1}, \mu_{2} \in \operatorname{Curr}\left(F_{N}\right)$, $c_{1}, c_{2} \geq 0$.

(2) $\langle c T, \mu\rangle=c\langle T, \mu\rangle$ for any $T \in \overline{\mathrm{cv}}_{N}, \mu \in \operatorname{Curr}\left(F_{N}\right)$ and $c \geq 0$.

(3) $\langle\phi T, \phi \mu\rangle=\langle T, \mu\rangle$ for any $T \in \overline{\mathrm{cv}}_{N}, \mu \in \operatorname{Curr}\left(F_{N}\right)$ and $\phi \in \operatorname{Out}\left(F_{N}\right)$.

(4) $\left\langle T, \eta_{g}\right\rangle=\|g\|_{T}$ for every $T \in \overline{\mathrm{cv}}_{N}$ and $g \in F_{N}, g \neq 1$.

The value $\langle T, \mu\rangle$ is called the geometric intersection number of $T \in \overline{\mathrm{cv}}_{N}$ and $\mu \in$ $\operatorname{Curr}\left(F_{N}\right)$.

\subsection{The minimal set}

Definition 4.4 (Minimal set $\mathbb{M}_{N}$ ) Let $N \geq 2$. Denote by $\mathbb{M}_{N}$ the closure in $\mathbb{P} \operatorname{Curr}\left(F_{N}\right)$ of the set

$$
\left\{\left[\eta_{g}\right]: g \in F_{N} \text { is primitive }\right\} \text {. }
$$


It is easy to see that $\mathbb{M}_{N} \subseteq \mathbb{P} \operatorname{Curr}\left(F_{N}\right)$ is a closed $\operatorname{Out}\left(F_{N}\right)$-invariant subset. It turns out (see Kapovich and Lustig [39]) that for $N \geq 3$ this is the minimal such subset:

Proposition 4.5 Let $N \geq 2$. Then:

(1) (Martin [50]) For every element $[\mu] \in \mathbb{M}_{N}$ the subset $\operatorname{Out}\left(F_{N}\right)[\mu]$ is dense in $\mathbb{M}_{N}$.

(2) (Kapovich-Lustig [39]) Let $N \geq 3$. Then $\mathbb{M}_{N} \subseteq \mathbb{P} \operatorname{Curr}\left(F_{N}\right)$ is the unique minimal closed $\operatorname{Out}\left(F_{N}\right)$-invariant nonempty subset. This means that whenever $Z \subseteq \mathbb{P} \operatorname{Curr}\left(F_{N}\right)$ is a closed $\operatorname{Out}\left(F_{N}\right)$-invariant nonempty subset then $\mathbb{M}_{N} \subseteq Z$.

For $N \geq 3$ part (1) of Proposition 4.5 follows directly from part (2). For $N=2$ part (1) of Proposition 4.5 follows from the results of Reiner Martin [50] who showed that $\mathbb{M}_{2}$ is homeomorphic to the circle and that the action of $\operatorname{Out}\left(F_{2}\right)=G L(2, \mathbb{Z})$ on $\mathbb{M}_{2}$ can be identified with the standard action of $G L(2, \mathbb{Z})$ on $\mathbb{S}^{1}=\partial H^{2}$.

The conclusion of part (2) of Proposition 4.5 is false for $N=2$. Indeed, for $F_{2}=$ $F(a, b)$, it is easy to see that $\eta_{g}$ for $g=[a, b]$ is a fixed point for the action of $\operatorname{Out}\left(F_{2}\right)$ on $\operatorname{Curr}\left(F_{2}\right)$ and hence $\left[\eta_{g}\right]$ is fixed by the action of $\operatorname{Out}\left(F_{2}\right)$ on $\mathbb{P} \operatorname{Curr}\left(F_{2}\right)$. However, we will see later that a weaker version of Proposition 4.5 is true for $N=2$ and that version will be sufficient for our purposes.

\subsection{Consequences of spectral rigidity of the set of primitive elements}

Recall that $\mathcal{P}_{N}$ denotes the set of all primitive elements in $F_{N}$.

A key tool in proving our main results is the following:

Proposition 4.6 Let $N \geq 2$, let $H \leq \operatorname{Aut}\left(F_{N}\right)$ and let $g \in F_{N}, g \neq 1$. Suppose that the closure of the set $H\left[\eta_{g}\right]$ in $\mathbb{P} \operatorname{Curr}\left(F_{N}\right)$ contains the set $\mathbb{M}_{N}$.

Then

$$
H g=\{\phi(g) \mid \phi \in H\} \subseteq F_{N}
$$

is a spectrally rigid subset of $F_{N}$.

Proof Let $T, T^{\prime} \in \mathrm{cv}_{N}$ be such that $\|\phi(g)\|_{T}=\|\phi(g)\|_{T^{\prime}}$ for every $\phi \in H$. We need to show that $T=T^{\prime}$ in $\mathrm{cv}_{N}$.

Let $Z$ be the closure of the set $H\left[\eta_{g}\right]$ in $\mathbb{P} \operatorname{Curr}\left(F_{N}\right)$. By assumption we have $\mathbb{M}_{N} \subseteq Z$. Hence for every primitive element $a \in F_{N}$ there exists a sequence $\phi_{n} \in H$ and a sequence $c_{n} \geq 0$ such that

$$
\lim _{n \rightarrow \infty} c_{n} \phi_{n} \eta_{g}=\lim _{n \rightarrow \infty} c_{n} \eta_{\phi_{n}(g)}=\eta_{a} \quad \text { in } \operatorname{Curr}\left(F_{N}\right)
$$


Proposition 4.3 then implies that

$$
\begin{gathered}
\|a\|_{T}=\left\langle T, \eta_{a}\right\rangle=\left\langle T, \lim _{n \rightarrow \infty} c_{n} \eta_{\phi_{n}(g)}\right\rangle=\lim _{n \rightarrow \infty} c_{n}\left\langle T, \eta_{\phi_{n}(g)}\right\rangle= \\
\lim _{n \rightarrow \infty} c_{n}\left\|\phi_{n}(g)\right\|_{T}=\lim _{n \rightarrow \infty} c_{n}\left\|\phi_{n}(g)\right\|_{T^{\prime}}=\lim _{n \rightarrow \infty} c_{n}\left\langle T^{\prime}, \eta_{\phi_{n}(g)}\right\rangle= \\
\left\langle T^{\prime}, \lim _{n \rightarrow \infty} c_{n} \eta_{\phi_{n}(g)}\right\rangle=\left\langle T^{\prime}, \eta_{a}\right\rangle=\|a\|_{T^{\prime}} .
\end{gathered}
$$

Thus $\|a\|_{T}=\|a\|_{T^{\prime}}$ for every primitive element $a \in F_{N}$. Theorem 3.4 now implies that $T=T^{\prime}$ in $\mathrm{cv}_{N}$.

\subsection{Stable and unstable currents}

Let $N \geq 2$. An element $\phi \in \operatorname{Out}\left(F_{N}\right)$ is called fully irreducible or iwip (for "irreducible with irreducible powers") if there is no integer $n \geq 1$ such that $\phi^{n}$ preserves the conjugacy class of a proper free factor of $F_{N}$.

For an element $\phi \in \operatorname{Out}\left(F_{N}\right)$ the conjugacy class $[g]$, where $g \in F_{N}, g \neq 1$, is periodic if there exists $n \geq 1$ such that $\phi^{n}[g]=[g]$. An element $\phi \in \operatorname{Out}\left(F_{N}\right)$ is called atoroidal if $\phi$ does not have any periodic conjugacy classes.

It is well-known that all non-atoroidal iwips in $\operatorname{Out}\left(F_{N}\right)$ come from homeomorphisms of compact surfaces with a single boundary component:

Proposition 4.7 (Bestvina and Handel [5]) Let $N \geq 2$ and $\phi \in \operatorname{Out}\left(F_{N}\right)$ be an iwip. Then the following hold:

(1) The automorphism $\phi$ is not atoroidal if and only if there exists an isomorphism $\alpha: F_{N} \rightarrow \pi_{1}(\Sigma)$, where $\Sigma$ is a connected compact surface with exactly one boundary component, such that $\phi$ is induced by a homeomorphism of $\Sigma$.

(2) Let $\phi \in \operatorname{Out}\left(F_{N}\right)$ be a non-atoroidal iwip, let $\Sigma$ be as in (1) and let [h] be the conjugacy class in $F_{N}$ given by the boundary of $\Sigma$.

Then the only periodic conjugacy classes of $\phi$ in $F_{N}$ are those of the form $\left[h^{m}\right]$, where $m \in \mathbb{Z}, m \neq 0$. The conjugacy class $[h]$ is called the peripheral curve of $\phi$. (Note that for an non-atoroidal iwip $\phi$ the peripheral curve $[h]$ is defined uniquely up to inversion. Namely, if $[g]$ is a periodic conjugacy class of $\phi$ such that $g \in F_{N}$ is not a proper power then $[g]=\left[h^{ \pm 1}\right]$.)

(3) Let $N=2$ and $F_{2}=F(a, b)$. Let $u=[a, b] \in F_{2}$. Let $\phi \in \operatorname{Out}\left(F_{2}\right)$ be an iwip. Then $\phi$ is not atoroidal and $[u]$ is the peripheral curve of $\phi$.

For an element $\phi \in \operatorname{Out}\left(F_{N}\right)$ a current $\mu \in \operatorname{Curr}\left(F_{N}\right)$ is called an eigencurrent of $\phi$ if $\mu \neq 0$ and $\phi \mu=\lambda \mu$ for some $\lambda \geq 0$. In that case the number $\lambda$ is called the 
associated eigenvalue of $\mu$ for $\phi$. Thus for $\mu \in \operatorname{Curr}\left(F_{N}\right), \mu \neq 0$ is an eigencurrent of $\phi$ if and only if $[\mu] \in \mathbb{P} \operatorname{Curr}\left(F_{N}\right)$ is a fixed point of $\phi$. If $[\mu] \in \mathbb{P} \operatorname{Curr}\left(F_{N}\right)$ is a fixed point of $\phi$, we also refer to the eigenvalue of $\mu$ for $\phi$ as the eigenvalue of $[\mu]$ for $\phi$, and we sometimes refer to $[\mu]$ as an eigencurrent of $\phi$.

Recall that $\mathbb{M}_{N} \subseteq \mathbb{P} \operatorname{Curr}\left(F_{N}\right)$ is a closed Out $\left(F_{N}\right)$-invariant subset. As proved by Reiner Martin [50], if $\phi \in \operatorname{Out}\left(F_{N}\right)$ is an iwip, then $\phi$ has the "North-South" dynamics on the minimal set $\mathbb{M}_{N} \subseteq \mathbb{P} \operatorname{Curr}\left(F_{N}\right)$ and, moreover, if $\phi$ is an atoroidal iwip, then $\phi$ has the "North-South" dynamics on $\mathbb{P} \operatorname{Curr}\left(F_{N}\right)$. We only need the following weak version of Martin's result:

Proposition 4.8 (Stable and unstable eigencurrents of iwips. Martin [50]) Let $N \geq 2$ and let $\phi \in \operatorname{Out}\left(F_{N}\right)$ be an iwip.

Then the following holds:

(1) The element $\phi$ has exactly two distinct fixed points in $\mathbb{M}_{N}$. One of these fixed points, called the stable eigencurrent of $\phi$, and denoted by $\left[\mu_{+}\right]=\left[\mu_{+}(\phi)\right] \in$ $\mathbb{M}_{N}$, has eigenvalue $>1$ for $\phi$, and the other fixed point, called the unstable eigencurrent of $\phi$ and denoted by $\left[\mu_{-}\right]=\left[\mu_{-}(\phi)\right] \in \mathbb{M}_{N}$, has eigenvalue $<1$ for $\phi$. Thus $\phi \mu_{+}=\lambda_{+} \mu_{+}$for $\lambda_{+}>1$ and $\phi \mu_{-}=\frac{1}{\lambda_{-}} \mu_{-}$for $\lambda_{-}>1$.

(2) If $\phi$ is both atoroidal and an iwip then for every $g \in F_{N}, g \neq 1$ we have

$$
\lim _{n \rightarrow \infty} \phi^{n}\left[\eta_{g}\right]=\left[\mu_{+}(\phi)\right], \quad \lim _{n \rightarrow \infty} \phi^{-n}\left[\eta_{g}\right]=\left[\mu_{-}(\phi)\right]
$$

(3) If $\phi$ is an iwip which is not atoroidal and if $[u]$ is the peripheral curve for $\phi$ then for every nontrivial $g \in F_{N}$ such that $g$ is not conjugate to $u^{m}, m \in \mathbb{Z}$ we have

$$
\lim _{n \rightarrow \infty} \phi^{n}\left[\eta_{g}\right]=\left[\mu_{+}(\phi)\right], \quad \lim _{n \rightarrow \infty} \phi^{-n}\left[\eta_{g}\right]=\left[\mu_{-}(\phi)\right] .
$$

(4) For any $\theta \in \operatorname{Out}\left(F_{N}\right)$ the element $\phi^{\prime}:=\theta \phi \theta^{-1}$ is again an iwip and $\left[\mu_{+}\left(\phi^{\prime}\right)\right]=$ $\theta\left[\mu_{+}(\phi)\right],\left[\mu_{-}\left(\phi^{\prime}\right)\right]=\theta\left[\mu_{-}(\phi)\right]$.

\section{Spectrally rigid automorphic orbits}

\subsection{Finding an iwip in an ample subgroup}

Lemma 5.1 Let $N \geq 2$ and let $H \leq \operatorname{Out}\left(F_{N}\right)$ be an infinite normal subgroup. Then $H$ contains an iwip element $\phi$. 
Proof By a result of Handel and Mosher [33] either $H$ contains an iwip or there exist a subgroup $H_{1}$ of finite index in $H$ and a proper free factor $B$ of $F_{N}$ such that every element of $H_{1}$ leaves the conjugacy class of $B$ invariant.

Suppose that the latter case occurs, so that some subgroup $H_{1}$ of finite index in $H$ preserves the conjugacy class of a proper free factor $B$ of $F_{N}$.

It is well known (see, for example, Bridson and Vogtmann [9]) that $\operatorname{Out}\left(F_{N}\right)$ is virtually torsion-free and hence there are no infinite torsion subgroups in $\operatorname{Out}\left(F_{N}\right)$. Thus if we show that every element of $H_{1}$ has finite order, this will imply that $H_{1}$ is finite, yielding a contradiction with the assumption that $H$ is infinite and that $H_{1}$ has finite index in $H$.

Note that if $\theta \in \operatorname{Out}\left(F_{N}\right)$ is arbitrary, then $\theta H_{1} \theta^{-1}$ leaves the conjugacy class $[\theta(B)]$ invariant. Since $H$ is normal in $\operatorname{Out}\left(F_{N}\right)$, it follows that $\theta H_{1} \theta^{-1}$ has finite index in $\theta H \theta^{-1}=H$ and hence $H_{\theta}:=H_{1} \cap \theta H_{1} \theta^{-1}$ has finite index in $H_{1}$. Thus every element of $H_{1}$ has a positive power belonging to $H_{\theta}$. Similarly, for any finite collection of elements $\theta_{1}, \ldots, \theta_{m} \in \operatorname{Out}\left(F_{N}\right)$ the subgroup

$$
H_{\theta_{1}, \ldots, \theta_{m}}:=H_{1} \cap \theta_{1} H_{1} \theta_{1}^{-1} \cap \ldots \cap \theta_{m} H_{1} \theta_{m}^{-1}
$$

has finite index in $H_{1}$. Moreover, every element of $H_{\theta_{1}, \ldots, \theta_{m}}$ leaves invariant each of the conjugacy classes $[B],\left[\theta_{1}(B)\right], \ldots\left[\theta_{m}(B)\right]$ and every element of $H_{1}$ has a positive power that belongs to $H_{\theta_{1}, \ldots, \theta_{m}}$.

Let $\psi \in H_{1}$ be arbitrary. Choose a free basis $A=\left\{a_{1}, \ldots, a_{N}\right\}$ of $F_{N}$ such that for some $1 \leq k \leq N-1$ the set $\left\{a_{1}, \ldots, a_{k}\right\}$ is a free basis of $B$. Let $\Psi \in \operatorname{Aut}\left(F_{N}\right)$ be a lift of $\psi$ to $\operatorname{Out}\left(F_{N}\right)$. Since $\psi$ preserves the conjugacy class of $B$, for every $n \in \mathbb{Z}$ the cyclically reduced form of $\Psi^{n}\left(a_{1}\right)$ over $A$ does not involve $a_{N}^{ \pm 1}$. For each $m=2, \ldots, N-1$ let $\theta_{m}$ be the automorphism of $F_{N}$ induced by the permutation of $A$ which interchanges $a_{m}$ and $a_{N}$ and leaves the other elements of $A$ fixed. Thus $\theta_{m}(B)$ is generated by a subset of $A$ that does not involve $a_{m}$. Let $n \geq 1$ be such that $\Psi^{n}$ belongs to $H_{\theta_{2}, \ldots, \theta_{N-1}}$. Then the image $\Psi^{n}\left(a_{1}\right)$ is a freely reduced word over $A$ whose cyclically reduced form does not involve $a_{2}^{ \pm 1}, \ldots, a_{N}^{ \pm 1}$, so that $\Psi\left(a_{1}\right)$ is conjugate to $a_{1}^{ \pm 1}$ in $F_{N}$. By taking a larger finite collection of automorphisms $\theta$ of $F_{N}$ we can find a positive power $\Psi$ which sends every $a_{i}$ to a conjugate of $a_{i}$ in $F_{N}$, where $i=1, \ldots, N$.

Moreover, by considering the automorphisms $\theta$ of $F_{N}$ corresponding to all the elementary Nielsen transformations on $A$, we can find an even bigger positive power $\Psi^{M}$ of $\Psi$ with the property that $\Psi^{M}(u)$ is conjugate to $u$ in $F_{N}$ for every freely reduced word $u$ of length $\leq 2$ over $A$. It is well known and easy to see that this 
implies that $\Psi^{M}$ is an inner automorphism of $F_{N}$, so that $\psi^{M}=1 \operatorname{in} \operatorname{Out}\left(F_{N}\right)$. Thus we have shown that every element of $H_{1}$ has finite order in $\operatorname{Out}\left(F_{N}\right)$, which, since $\operatorname{Out}\left(F_{N}\right)$ is virtually torsion-free, implies that $H_{1}$ is finite. However, this contradicts the assumption that $H$ is infinite and that $H_{1}$ has finite index in $H$.

Thus $H$ contains an iwip element, as required.

Remark 5.2 Note that for $N=2$ every automorphism of $F_{2}=F(a, b)$ has a periodic conjugacy class - namely the conjugacy class of the commutator $[a, b]$. Thus every iwip $\phi \in \operatorname{Out}\left(F_{2}\right)$ is toroidal.

For $N \geq 3$ it is almost certainly the case that every nontrivial normal subgroup $H$ of $\operatorname{Out}\left(F_{N}\right)$ contains an atoroidal iwip (and not just an iwip, as Lemma 5.1 proves). Such a strengthening of Lemma 5.1 would eliminate the need to consider Case 2 in the proof of Theorem 5.4 below. However, the presently available tools do not appear to be sufficient for establishing the existence of an atoroidal iwip in $H$. Let $\phi \in H$ be an iwip whose existence is provided by Lemma 5.1. Suppose that $\phi$ is toroidal. We can use then ping-pong considerations for the action of $\operatorname{Out}\left(F_{N}\right)$ on the free factor complex $F F_{N}$ (now known to be Gromov-hyperbolic by a result of Bestvina and Feighn [1])) and choose a conjugate $\psi=\theta \phi \theta^{-1} \in H$ such that for all sufficiently large $n \geq 1$ the subgroup $\Gamma=\left\langle\phi^{n}, \psi^{n}\right\rangle \leq H$ is free of rank two and that every nontrivial element of $\Gamma$ is again an iwip. One would then like to argue that (with the appropriate choices of $\theta$ and $n$ ) the element $\phi^{n} \psi^{n} \in H$ is an atoroidal iwip. The difficulty in proving this statement is that toroidal iwips do not act with "north-south" dynamics on $\mathbb{P} \operatorname{Curr}\left(F_{N}\right)$. Instead, a toroidal iwip $\beta$ has three distinct fixed points in $\mathbb{P} \operatorname{Curr}\left(F_{N}\right)$ : the stable (expanding) current $\left[\mu_{+}(\beta)\right]$, the unstable (contracting) current $\left[\mu_{-}(\beta)\right]$ and the current $\left[\eta_{u}\right]$ (where $u$ is the peripheral curve for $\beta$ ) such that $\eta_{u}$ is fixed by $\beta$ in the non-projective sense. To prove that the iwip $\alpha=\phi^{n} \psi^{n}$ is atoroidal we need to show that there does not exist a nontrivial current in $\operatorname{Curr}\left(F_{N}\right)$ that is fixed by $\alpha$. Establishing this fact requires first proving the following generalized north-south dynamical property for the action of any toroidal iwip $\beta \in \operatorname{Out}\left(F_{N}\right)$ for $N \geq 3$ : for any neighborhood $U$ of $\left[\mu_{+}(\beta)\right]$ in $\mathbb{P} \operatorname{Curr}\left(F_{N}\right)$ and for any open set $V$ in $\mathbb{P} \operatorname{Curr}\left(F_{N}\right)$ containing the segment between $\left[\mu_{-}(\beta)\right]$ and $\left[\eta_{u}\right]$ (where $u$ is the peripheral curve for $\beta$ ) there exists $M \geq 1$ such that for all $m \geq M$ we have $\beta^{m}\left(\mathbb{P} \operatorname{Curr}\left(F_{N}\right) \backslash V\right) \subseteq U$. This statement, which is most likely true, is not yet proved in the literature and establishing it requires different arguments than those used by Reiner Martin [50] in the proof of "north-south" dynamics for the action of atoroidal iwips on $\mathbb{P} \operatorname{Curr}\left(F_{N}\right)$. 


\subsection{The proof of Theorem $A$ for the case $N \geq 3$}

Lemma 5.3 Let $N \geq 3$ and let $H \leq \operatorname{Out}\left(F_{N}\right)$ be an infinite normal subgroup. Then for every nontrivial element $g \in F_{N}$ there exists $\psi \in H$ such that $\psi(g)$ is not conjugate to $g^{ \pm 1}$ in $F_{N}$.

Proof Let $g \in F_{N}, g \neq 1$. Suppose that for every $\psi \in H \psi(g)$ is conjugate to $g$ or $g^{-1}$ in $F_{N}$. Thus $H\left[\eta_{g}\right]=\left[\eta_{g}\right]$. Hence for every $\theta \in \operatorname{Out}\left(F_{N}\right)$ the subgroup $\theta H \theta^{-1}$ fixes $\theta\left[\eta_{g}\right]=\left[\eta_{\theta(g)}\right]$ in $\mathbb{P} \operatorname{Curr}\left(F_{N}\right)$. Since $H$ is normal in $\operatorname{Out}\left(F_{N}\right)$, it follows that $H$ fixes $\theta\left[\eta_{g}\right]=\left[\eta_{\theta(g)}\right]$ for every $\theta \in \operatorname{Out}\left(F_{N}\right)$.

Let $\varphi \in \operatorname{Out}\left(F_{N}\right)$ be any atoroidal iwip. Then $H$ fixes $\varphi^{n}\left[\eta_{g}\right]$ for every $n \geq 1$. Hence, by Proposition 4.8, we have $H\left[\mu_{+}(\varphi)\right]=\left[\mu_{+}(\varphi)\right]$. By a result of Kapovich and Lustig [43], for any atoroidal iwip $\varphi$ the stabilizer of $\left[\mu_{+}(\varphi)\right]$ in $\operatorname{Out}\left(F_{N}\right)$ is virtually cyclic and contains $\langle\varphi\rangle$ as a subgroup of finite index. Now choose two atoroidal iwips $\varphi_{1}, \varphi_{2} \in \operatorname{Out}\left(F_{N}\right)$ so that $\left\langle\varphi_{1}\right\rangle \cap\left\langle\varphi_{1}\right\rangle=\{1\}$. Then, by the above argument, the intersection of the stabilizers of $\left[\mu_{+}\left(\varphi_{1}\right)\right]$ and $\left[\mu_{+}\left(\varphi_{2}\right)\right]$ must be finite and must contain $H$, which contradicts the assumption that $H$ is an infinite subgroup of $\operatorname{Out}\left(F_{N}\right)$.

The following statement implies Theorem A from the Introduction for the case $N \geq 3$ :

Theorem 5.4 Let $N \geq 3$. Let $H \leq \operatorname{Aut}\left(F_{N}\right)$ be an ample subgroup.

Then for every $g \in F_{N}, g \neq 1$, the automorphic orbit

$$
H g \subseteq F_{N}
$$

is a spectrally rigid subset of $F_{N}$.

Proof Denote by $\bar{H} \leq \operatorname{Out}\left(F_{N}\right)$ the image of $H$ in $\operatorname{Out}\left(F_{N}\right)$. Thus $\bar{H}$ is contains an infinite normal subgroup of $\operatorname{Out}\left(F_{N}\right)$, and, without loss of generality we may assume that $\bar{H}$ is an infinite normal subgroup of $\operatorname{Out}\left(F_{N}\right)$.

Let $g \in F_{N}, g \neq 1$ be arbitrary. Let $Z$ be the closure of the set $H\left[\eta_{g}\right]$ in $\mathbb{P} \operatorname{Curr}\left(F_{N}\right)$. Proposition 4.6 implies that in order to establish that $\mathrm{Hg}$ is a spectrally rigid subset of $F_{N}$ it suffices to establish the following:

Claim We have $\mathbb{M}_{N} \subseteq Z$.

Lemma 5.1 implies that $\bar{H}$ contains some iwip element $\phi$.

Case 1 Suppose first that the iwip $\phi$ is atoroidal.

By Proposition 4.8 we have

$$
\lim _{n \rightarrow \infty} \phi^{n}\left[\eta_{g}\right]=\left[\mu_{+}(\phi)\right]
$$


Therefore $\left[\mu_{+}(\phi)\right] \in Z$. Recall also that $\left[\mu_{+}(\phi)\right] \in \mathbb{M}_{N}$.

Let $\theta \in \operatorname{Out}\left(F_{N}\right)$ be arbitrary. Since $\bar{H}$ is normal in $\operatorname{Out}\left(F_{N}\right)$, it follows that $\theta \phi \theta^{-1} \in$ $\bar{H}$. The element $\phi^{\prime}=\theta \phi \theta^{-1}$ is again an iwip. Hence the same argument as above implies that $\mu_{+}\left(\theta \phi \theta^{-1}\right) \in Z$.

Since $\mu_{+}\left(\theta \phi \theta^{-1}\right)=\theta\left[\mu_{+}(\phi)\right]$, we see that $\theta\left[\mu_{+}(\phi)\right] \in Z$ for every $\theta \in \operatorname{Out}\left(F_{N}\right)$. By Proposition 4.5 the subset $\operatorname{Out}\left(F_{N}\right)\left[\mu_{+}(\phi)\right] \subseteq \mathbb{M}_{N}$ is dense in $\mathbb{M}_{N}$. Since $Z$ is closed, this implies that $\mathbb{M}_{N} \subseteq Z$, as claimed.

Case 2 Suppose that the iwip $\phi$ is not atoroidal. Let $[u]$ be the peripheral curve of $[\phi]$.

Since $H g=H \Psi(g)$ for every $\Psi \in H$, Lemma 5.3 implies that, after possibly replacing $g$ by $\Psi(g)$ for some $\Psi \in H$, we may assume that $\left[\eta_{g}\right] \neq\left[\eta_{u}\right]$. Then Proposition 4.8 implies that

$$
\lim _{n \rightarrow \infty} \phi^{n}\left[\eta_{g}\right]=\left[\mu_{+}(\phi)\right]
$$

Thus again we see that $\left[\mu_{+}(\phi)\right] \in Z$.

Let $\theta \in \operatorname{Out}\left(F_{N}\right)$ be arbitrary and let $\phi^{\prime}=\theta \phi \theta^{-1}$. Thus $\left[\mu_{+}\left(\phi^{\prime}\right)\right]=\theta\left[\mu_{+}(\phi)\right]$ and $\theta(u)$ is the peripheral curve of $\phi^{\prime}$.

If $\left[\eta_{g}\right] \neq\left[\eta_{\theta(u)}\right]$ then again Proposition 4.8 implies that $\lim _{n \rightarrow \infty}\left(\phi^{\prime}\right)^{n}\left[\eta_{g}\right]=\left[\mu_{+}\left(\phi^{\prime}\right)\right]=$ $\theta\left[\mu_{+}(\phi)\right]$, so that $\left[\mu_{+}\left(\phi^{\prime}\right)\right]=\theta\left[\mu_{+}(\phi)\right] \in Z$.

Suppose now that $\left[\eta_{g}\right]=\left[\eta_{\theta(u)}\right]$. Then Lemma 5.3 again implies that there is some $\psi \in \bar{H}$ such that $\psi\left[\eta_{g}\right]=\left[\eta_{\psi(g)}\right] \neq\left[\eta_{\theta(u)}\right]$. Note that $\bar{H}[g]=\bar{H}[\psi(g)]$ since $\psi \in \bar{H}$. Proposition 4.8 now implies that

$$
\lim _{n \rightarrow \infty}\left(\phi^{\prime}\right)^{n}\left[\eta_{\psi(g)}\right]=\left[\mu_{+}\left(\phi^{\prime}\right)\right] .
$$

Thus again $\left[\mu_{+}\left(\phi^{\prime}\right)\right]=\theta\left[\mu_{+}(\phi)\right] \in Z$.

We have shown that $\theta\left[\mu_{+}(\phi)\right] \in Z$ for every $\theta \in \operatorname{Out}\left(F_{N}\right)$. Since $\operatorname{Out}\left(F_{N}\right)\left[\mu_{+}(\phi)\right]$ is dense in $\mathbb{M}_{N}$ and the set $Z$ is closed, it follows that $\mathbb{M}_{N} \subseteq Z$.

Thus the Claim is verified, which, as noted above, via Proposition 4.6 now implies that $H g$ is a spectrally rigid subset of $F_{N}$.

\subsection{The case $N=2$}

Fix a free basis $A:=\{a, b\}$ of $F_{2}$, so that $F_{2}=F(a, b)$.

We need the following weaker version of Proposition 4.5 for the case $N=2$ : 
Proposition 5.5 Let $g \in F_{2}, g \neq 1$ be such that $g$ is not conjugate to any integer power of $[a, b]$. Let $H \leq \operatorname{Out}\left(F_{2}\right)$ be an infinite normal subgroup. Let $Z$ be any closed $H$-invariant subset of $\mathbb{P} \operatorname{Curr}\left(F_{2}\right)$ such that $\left[\eta_{g}\right] \in Z$. Then $\mathbb{M}_{2} \subseteq Z$.

Proof Let $Z$ be the closure of the set $H\left[\eta_{g}\right]=\left\{\left[\eta_{\phi(g)}\right]: \phi \in H\right\}$ in $\mathbb{P} \operatorname{Curr}\left(F_{2}\right)$.

Lemma 5.1 implies that there exists an iwip element $\phi \in H$. Recall that in this case $u=[a, b]$ is the peripheral curve for $\phi$ and, moreover, powers of $[a, b]$ are the only periodic conjugacy classes for $\phi$ in $F_{2}$.

Since by assumption $g$ is not conjugate to any integer power of $[a, b]$, it follows from Proposition 4.8 that

$$
\lim _{n \rightarrow \infty} \phi^{n}\left[\eta_{g}\right]=\lim _{n \rightarrow \infty}\left[\eta_{\phi^{n}(g)}\right]=\left[\mu_{+}(\phi)\right] .
$$

Therefore $\left[\mu_{+}(\phi)\right] \in Z$. For every $\theta \in \operatorname{Out}\left(F_{2}\right) \phi^{\prime}=\theta \phi \theta^{-1}$ is again an iwip with $\left[\mu_{+}\left(\phi^{\prime}\right)\right]=\theta\left[\mu_{+}(\phi)\right]$. The subgroup $H$ is normal in $\operatorname{Out}\left(F_{N}\right)$, and therefore $\phi^{\prime} \in H$. Now Proposition 4.8 again implies that $\left[\mu_{+}\left(\phi^{\prime}\right)\right] \in Z$. Since $\left[\mu_{+}\left(\phi^{\prime}\right)\right]=\theta\left[\mu_{+}(\phi)\right]$, we have shown that $\theta\left[\mu_{+}(\phi)\right] \in Z$ for every $\theta \in \operatorname{Out}\left(F_{2}\right)$. By Proposition 4.5, every $\operatorname{Out}\left(F_{2}\right)$-orbit of a point of $\mathbb{M}_{2}$ is dense in $\mathbb{M}_{2}$, and therefore $\mathbb{M}_{2} \subseteq Z$, as required.

Remark 5.6 Suppose $g$ is conjugate in $F_{2}=F(a, b)$ to $[a, b]^{k}$ for some $k \in \mathbb{Z}$. Then $\operatorname{Aut}\left(F_{2}\right) g \subseteq F_{2}$ is not spectrally rigid.

Indeed, let $T_{A}$ and $T_{B}$ be the Cayley graphs of $F(a, b)$ with respect to $A=\{a, b\}$ and $B=\{a, a b\}$ accordingly. As usual, we give all edges of $T_{A}, T_{B}$ length 1 . Then $T_{A}, T_{B} \in \mathrm{cv}_{2}$ and $T_{A} \neq T_{B}$ in $\mathrm{cv}_{2}$.

It is well-known that for any $\phi \in \operatorname{Aut}(F(a, b))$, the element $\phi([a, b])$ is conjugate to $[a, b]^{ \pm 1}$ in $F(a, b)$. It follows that for any free bases $A$ and $B$ of $F_{2}=F(a, b)$, and for any $\phi \in \operatorname{Aut}\left(F_{2}\right)$ we have $\|\phi(g)\|_{A}=\|\phi(g)\|_{B}=4|k|$. The Cayley graphs $T_{A}$ and $T_{B}$ of $F_{2}$ with respect to $A$ and $B$ respectively are both points in $\mathrm{cv}_{2}$. Thus we see that for every $\phi \in \operatorname{Aut}\left(F_{2}\right)$ we have $\|\phi(g)\|_{T_{A}}=\|\phi(g)\|_{T_{B}}=4|k|$. Since we chose $A$ and $B$ so that $T_{A} \neq T_{B}$ in $\mathrm{cv}_{2}$, this shows that the orbit $\operatorname{Aut}(F(a, b)) g$ is not spectrally rigid in $F(a, b)$.

It turns out that Remark 5.6 provides the only obstruction to extending Theorem 5.4 to the case $N=2$, and we obtain the conclusion of Theorem A from the Introduction for $N=2$ :

Theorem 5.7 Let $F_{2}=F(a, b)$ and let $g \in F_{2}, g \neq 1$ be such that $g$ is not conjugate to a power of $[a, b]$ in $F(a, b)$.

Let $H \leq \operatorname{Aut}\left(F_{2}\right)$ be an ample subgroup. Then $\mathrm{Hg}$ is a spectrally rigid subset of $F(a, b)$. 
Proof Suppose that $g \in F(a, b), g \neq 1$ is such that $g$ is not conjugate to a power of $[a, b]$ in $F(a, b)$. Let $Z$ be the closure in $\mathbb{P} \operatorname{Curr}\left(F_{N}\right)$ of the set $H\left[\eta_{g}\right]$. Proposition 5.5 implies that $\mathbb{M}_{2} \subseteq Z$. Proposition 4.6 now implies that $H g$ is a spectrally rigid subset of $F_{2}$.

\section{Open problems}

One can define a more restrictive notion of spectral rigidity than the one considered in this paper. Namely, call a subset $S \subseteq F_{N}$ strongly spectrally rigid if whenever $T, T^{\prime} \in \overline{\mathrm{cv}}_{N}$ are such that $\|g\|_{T}=\|g\|_{T^{\prime}}$ for every $g \in S$ then $T=T^{\prime}$ in $\overline{\mathrm{cv}}_{N}$.

Problem 6.1 For $N \geq 3$ is it true that every nontrivial $\operatorname{Aut}\left(F_{N}\right)$ orbit is strongly spectrally rigid in $F_{N}$ ? Is it true that for $N \geq 3$ the set $\mathcal{P}_{N}$ of all the primitive elements is strongly spectrally rigid in $F_{N}$ ?

In Kapovich [38] it is proved that for the nonbacktracking simple random walk on $F_{N}$ almost every trajectory of that walk gives a strongly spectrally rigid subset of $F_{N}$. However, the proofs of Theorem 5.4 and Theorem 5.7 in the present paper do not imply strong spectral rigidity, primarily because our proof of spectral rigidity of the set $\mathcal{P}_{N}$ in Theorem 3.4 only works for the interior points of the Outer space. It was recently pointed out to us by Jing Tao that for $N=2$ the set $\mathcal{P}_{2}$ of all primitive elements in $F_{2}=F(a, b)$ is not strongly spectrally rigid in $F_{2}$. She provided the following example demonstrating this fact:

Example 6.2 (Jing Tao's example) Consider a graph of groups $\mathbb{A}$ with the underlying graph $A$ consisting of two vertices $x_{0}, x_{1}$ and two distinct topological edges $e$ and $f$, each with end-vertices $x_{0}, x_{1}$. Thus $A$ is a topological circle subdivided in two edges. Orient $e$ and $f$ so that both $e$ and $f$ have $x_{0}$ as the initial vertex and $x_{1}$ as the terminal vertex. Define the vertex and edge groups as $A_{x_{0}}=A_{x_{1}}=A_{e}=\langle a\rangle$ and $A_{f}=\{1\}$, with the boundary monomorphisms for the edge $e$ being the identity map $\langle a\rangle \rightarrow\langle a\rangle$. Identify $b$ with the loop $e f^{-1}$. This provides an identification between $F(a, b)$ and $\pi_{1}\left(\mathbb{A}, x_{0}\right)$. Note that the graph of groups $\mathbb{A}$ gives a very small simplicial splitting of $F(a, b)$. Finally, for each $t \in(0,1)$ give the edge $e$ length $t$ and the edge $f$ length $1-t$. Thus for every $t \in(0,1)$ we get a point $T_{t} \in \overline{\mathrm{cv}}_{2}$ given by the Bass-Serre tree of $\mathbb{A}$ with the lifts of $e$ having length $t$ and the lifts of $f$ having length $1-t$. By construction, for any distinct $t, t^{\prime} \in(0,1)$ we have $T_{t} \neq T_{t^{\prime}}$ in $\overline{\mathrm{cv}}_{2}$, and, moreover, the projective classes of $T_{t}$ and $T_{t^{\prime}}$ are also distinct. It is easy to check that for every $t \in(0,1)$ and every cyclically reduced word $w \in F_{2}$, where all $b$ s occur with the same 
sign, the translation length $\|w\|_{T_{t}}$ is equal to the absolute value of the exponent sum on $b$ in $w$. Since every primitive element in $F(a, b)$ has cyclically reduced form where all occurrences of $a$ have the same sign and all occurrences of $b$ have the same sign (see for example Cohen, Metzler and Zimmermann [14]), it follows that for any primitive element $w$ in $F(a, b)$ the translation length $\|w\|_{T_{t}}$ is a constant function of $t$. Thus the restriction of $\|\cdot\|_{T_{t}}$ to $\mathcal{P}_{2}$ is a function that does not vary with $t$, which shows that the set $\mathcal{P}_{2}$ is not strongly spectrally rigid. On the other hand, for every $t \in(0,1)$ we have $\|[a, b]\|_{T_{t}}=2 t-2$, so that knowing of the translation length of $[a, b]$ is already sufficient to distinguish $T_{t}$ from all the other trees in the family. As noted by the referee, one can also obtain this example geometrically as follows: Identify $F_{2}$ with the fundamental group $\pi_{1}(S)$ of a torus with a single boundary component, and put a hyperbolic metric on $S$ making the boundary component totally geodesic. Consider a measured geodesic lamination $(L, \mu)$ on $S$ consisting of one closed geodesic (say the meridian curve), of weight $t$, and a geodesic arc with two endpoints on the boundary, of weight $1-t$. Then the tree dual to the lift of this measured lamination to the universal cover $\widetilde{S}$ of $S$ is exactly the tree $T_{t}$ described above.

It seems plausible, however, that the case of $F_{2}$ is special (because of the rather special nature of primitive elements in $F_{2}$ ) and that for $N \geq 3$ the set $\mathcal{P}_{N}$ is strongly spectrally rigid in $F_{N}$. Some positive evidence in this direction is provided by the following observation. Let $\phi \in \operatorname{Out}\left(F_{N}\right)$ (where $N \geq 3$ ) be an atoroidal iwip element and let $T_{\phi} \in \mathrm{cv}_{N}$ be the "stable tree" of an atoroidal iwip $\phi \in \operatorname{Out}\left(F_{N}\right)$ (in particular $T_{\phi} \phi=$ $\lambda T_{\phi}$, where $\lambda>1$ is the Perron-Frobenius eigenvalue of a train-track representative of $\phi$ ). We can prove that whenever $T^{\prime} \in \mathrm{cv}_{N}$ is such that the lengths functions of $T_{\phi}$ and of $T^{\prime}$ agree on all primitive elements of $F_{N}$ then $T^{\prime}=T_{\phi}$ in $\overline{\mathrm{cv}}_{N}$. Namely, in this case one can show that the Bestvina-Feighn-Handel "legal" lamination $L_{B F H}(\phi)$ of $\phi$ is contained in the dual algebraic lamination $L^{2}\left(T^{\prime}\right)$ of $T^{\prime}$. This implies, for instance by the results of Kapovich and Lustig [42], that $\left[T_{\phi}\right]=\left[T^{\prime}\right]$ in $\overline{\mathrm{CV}}_{N}$, and it is then not hard to deduce that $T^{\prime}=T_{\phi}$. We refer the reader to Bestvina, Feighn and Handel [4], Kapovich and Lustig [42] and Coulbois, Hilion and Lustig [15; 16] for the background on dual algebraic laminations and on laminations associated to iwip automorphisms.

Problem 6.3 Let $N \geq 3$. Does there exist a subset $S \subseteq F_{N}$ such that $S$ is spectrally rigid but not strongly spectrally rigid?

Problem 6.4 Let $N \geq 2$ and let $S \subseteq \mathcal{P}_{N}$ be an arbitrary subset of the set $\mathcal{P}_{N}$ of all primitive elements in $F_{N}$. Since $S$ consists of primitive elements, this implies that $\left\{\left[\eta_{g}\right]: g \in S\right\} \subseteq \mathbb{M}_{N}$. 
Is it true that $S$ is spectrally rigid in $F_{N}$ if and only if the closure of $\left\{\left[\eta_{g}\right]: g \in S\right\}$ in $\mathbb{P} \operatorname{Curr}\left(F_{N}\right)$ is equal to $\mathbb{M}_{N}$ ?

It is easy to show, using the intersection form, that if the closure of $\left\{\left[\eta_{g}\right]: g \in S\right\}$ in $\mathbb{P} \operatorname{Curr}\left(F_{N}\right)$ is equal to $\mathbb{M}_{N}$ then $S$ is spectrally rigid. However, the converse implication appears to be quite difficult. A recent result of Duchin, Leininger and Rafi [26] establishes a similar statement to that suggested in Problem 6.4 in the context of singular flat metrics on surfaces.

We have seen in Theorem 5.4 that for $N \geq 3$ if $H \leq \operatorname{Aut}\left(F_{N}\right)$ is a subgroup that projects to an infinite normal subgroup of $\operatorname{Out}\left(F_{N}\right)$ then the $\operatorname{Aut}\left(F_{N}\right)$-orbit of every nontrivial element $g$ of $F_{N}$ is spectrally rigid. In an earlier version of this paper we conjectured that for every cyclic subgroup $H \leq \operatorname{Aut}\left(F_{N}\right)$ and every $g \in F_{N}$ the orbit $H g \subseteq F_{N}$ is not spectrally rigid in $F_{N}$. This conjecture was recently proved by Brian Ray [53]. This fact and Theorem 5.4 suggest the following:

Problem 6.5 Let $N \geq 3$ and let $H \leq \operatorname{Aut}\left(F_{N}\right)$ be an arbitrary subgroup. Is it true that either for every nontrivial $g \in F_{N}$ the orbit $H g \subseteq F_{N}$ is spectrally rigid or that for every nontrivial $g \in F_{N}$ the orbit $H g \subseteq F_{N}$ is not spectrally rigid?

A positive answer to the above question would mean that rigidity or non-rigidity of the orbit $H g$ (where $g \in F_{N}, g \neq 1$ ) depends only on the subgroup $H \leq \operatorname{Aut}\left(F_{N}\right)$ and not on the choice of a nontrivial element $g \in F_{N}$.

Theorem B motivates the following question:

Problem 6.6 (Relatively strongly rigid finite sets) Given $T \in \overline{\mathrm{cv}}_{N}$, does there exist a finite subset $S \subseteq \mathcal{P}_{N}$ such that whenever $T^{\prime} \in \overline{\mathrm{cv}}_{N}$ is such that $\|g\|_{T}=\|g\|_{T^{\prime}}$ for all $g \in S$ then $T=T^{\prime}$ in $\overline{\mathrm{cv}}_{N}$ ? What if we just require $S$ to be a finite subset of $F_{N}$ (and not necessarily of $\mathcal{P}_{N}$ )?

As we noted in the introduction, by a result of Cohen-Lustig-Steiner [13], there does not exist a finite spectrally rigid subset of $F_{2}$. However, the argument in [13] involves looking at trees $T \in \mathrm{cv}_{2}$ with variable volume of the quotient metric graph $T / F_{2}$. On the other hand, the Smillie-Vogtmann construction (see Culler and Vogtmann [23]) for $N \geq 3$ only uses trees with co-volume 1 , that is, points of $\mathrm{CV}_{N}$. In fact, for $N=2$ the situation is quite different when restricting trees with quotient graphs of volume 1, that is, to $\mathrm{CV}_{2}$. Elaborating the arguments from Culler and Vogtmann [24] we can show that for $F_{2}=F(a, b)$ the set $S_{0}:=\left\{a, b, a b, a b^{-1},[a, b]\right\}$ is " $\mathrm{CV}_{2}$-rigid", that is, knowing the $\|\cdot\|_{T}$-lengths, for an arbitrary $T \in \mathrm{CV}_{2}$, of elements of $S_{0}$, uniquely determines $T$. This naturally leads to the following question: 
Problem 6.7 Does there exist a finite $\mathrm{CV}_{2}$-rigid set of primitive elements in $F_{2}$ ?

The notion of a spectrally rigid set naturally generalizes to the space of currents. Thus we say that a subset $S \subseteq \operatorname{Curr}\left(F_{N}\right)$ is spectrally rigid if whenever $T, T^{\prime} \in \mathrm{cv}_{N}$ are such that $\langle T, \mu\rangle=\left\langle T^{\prime}, \mu\right\rangle$ for every $\mu \in S$ then $T=T^{\prime}$ in $\mathrm{cv}_{N}$.

Problem 6.8 Let $N \geq 2$. Does there exist a finite spectrally rigid set of currents $S \subseteq \operatorname{Curr}\left(F_{N}\right)$ ?

The original argument of Smillie-Vogtmann [54] about non-existence of a finite spectrally rigid set of elements in $F_{N}$, and the above mentioned result of Brian Ray [53] about non-rigidity of orbits of cyclic subgroups of $\operatorname{Aut}\left(F_{N}\right)$ significantly rely on the fact that (in the language of currents) counting currents of elements of $F_{N}$ never have full support. Thus it is possible that there may indeed exist a finite spectrally rigid set of currents containing one or more current with full support. On the other hand, it also seems plausible that every finite subset $S \subseteq \mathbb{M}_{N}$ is not spectrally rigid.

\section{References}

[1] M Bestvina, M Feighn, Hyperbolicity of the complex of free factors arXiv: 1107.3308

[2] M Bestvina, M Feighn, Outer limits, preprint (1993) Available at http:// andromeda.rutgers. edu/ feighn/papers/outer.pdf

[3] M Bestvina, M Feighn, A hyperbolic Out $\left(F_{n}\right)$-complex, Groups Geom. Dyn. 4 (2010) 31-58 MR2566300

[4] M Bestvina, M Feighn, M Handel, Laminations, trees, and irreducible automorphisms of free groups, Geom. Funct. Anal. 7 (1997) 215-244 MR1445386

[5] M Bestvina, M Handel, Train tracks and automorphisms of free groups, Ann. of Math. 135 (1992) 1-51 MR1147956

[6] F Bonahon, Bouts des variétés hyperboliques de dimension 3, Ann. of Math. 124 (1986) 71-158 MR847953

[7] F Bonahon, The geometry of Teichmüller space via geodesic currents, Invent. Math. 92 (1988) 139-162 MR931208

[8] M R Bridson, K Vogtmann, The symmetries of outer space, Duke Math. J. 106 (2001) 391-409 MR1813435

[9] M R Bridson, K Vogtmann, Automorphism groups of free groups, surface groups and free abelian groups, from: "Problems on mapping class groups and related topics", Proc. Sympos. Pure Math. 74, Amer. Math. Soc., Providence, RI (2006) 301-316 MR2264548 
[10] I Chiswell, Introduction to $\Lambda$-trees, World Scientific Publishing Co., River Edge, NJ (2001) MR1851337

[11] M Clay, A Pettet, Currents twisting and nonsingular matrices, Comment. Math. Helv. (to appear)

[12] M M Cohen, M Lustig, Very small group actions on $\mathbb{R}$-trees and Dehn twist automorphisms, Topology 34 (1995) 575-617 MR1341810

[13] M M Cohen, M Lustig, M Steiner, $\mathbb{R}$-tree actions are not determined by the translation lengths of finitely many elements, from: "Arboreal group theory (Berkeley, CA, 1988)", Math. Sci. Res. Inst. Publ. 19, Springer, New York (1991) 183-187 MR1105334

[14] M Cohen, W Metzler, A Zimmermann, What does a basis of $F(a, b)$ look like?, Math. Ann. 257 (1981) 435-445 MR639577

[15] T Coulbois, A Hilion, M Lustig, $\mathbb{R}$-trees and laminations for free groups I: Algebraic laminations, J. Lond. Math. Soc. 78 (2008) 723-736 MR2456901

[16] T Coulbois, A Hilion, M Lustig, $\mathbb{R}$-trees and laminations for free groups II: The dual lamination of an $\mathbb{R}$-tree, J. Lond. Math. Soc. 78 (2008) 737-754 MR2456902

[17] C B Croke, Rigidity for surfaces of nonpositive curvature, Comment. Math. Helv. 65 (1990) 150-169 MR1036134

[18] C B Croke, Rigidity theorems in Riemannian geometry, from: "Geometric methods in inverse problems and PDE control”, IMA Vol. Math. Appl. 137, Springer, New York (2004) 47-72 MR2169902

[19] C B Croke, P Eberlein, B Kleiner, Conjugacy and rigidity for nonpositively curved manifolds of higher rank, Topology 35 (1996) 273-286 MR1380497

[20] C Croke, A Fathi, J Feldman, The marked length-spectrum of a surface of nonpositive curvature, Topology 31 (1992) 847-855 MR1191384

[21] M Culler, Finite groups of outer automorphisms of a free group, from: "Contributions to group theory”, Contemp. Math. 33, Amer. Math. Soc., Providence, RI (1984) 197-207 MR767107

[22] M Culler, J W Morgan, Group actions on $\mathbb{R}$-trees, Proc. London Math. Soc. 55 (1987) 571-604 MR907233

[23] M Culler, K Vogtmann, Moduli of graphs and automorphisms of free groups, Invent. Math. 84 (1986) 91-119 MR830040

[24] M Culler, K Vogtmann, The boundary of outer space in rank two, from: "Arboreal group theory (Berkeley, CA, 1988)”, Math. Sci. Res. Inst. Publ. 19, Springer, New York (1991) 189-230 MR1105335

[25] F Dal'Bo, I Kim, Marked length rigidity for symmetric spaces, Comment. Math. Helv. 77 (2002) 399-407 MR1915048

[26] M Duchin, C J Leininger, K Rafi, Length spectra and degeneration of flat metrics, Invent. Math. 182 (2010) 231-277 MR2729268 
[27] A Fathi, F Laudenbach, V Poenaru, Travaux de Thurston sur les surfaces, Astérisque 66, Société Mathématique de France, Paris (1979) MR568308 Séminaire Orsay, With an English summary

[28] S Francaviglia, Geodesic currents and length compactness for automorphisms of free groups, Trans. Amer. Math. Soc. 361 (2009) 161-176 MR2439402

[29] S Francaviglia, A Martino, Metric properties of outer space, Publ. Mat. 55 (2011) 433-473 MR2839451

[30] V Guirardel, Approximations of stable actions on $\mathbb{R}$-trees, Comment. Math. Helv. 73 (1998) 89-121 MR1610591

[31] V Guirardel, Dynamics of $\operatorname{Out}\left(F_{n}\right)$ on the boundary of outer space, Ann. Sci. École Norm. Sup. 33 (2000) 433-465 MR1832819

[32] U Hamenstädt, Invariant Radon measures on measured lamination space, Invent. Math. 176 (2009) 223-273 MR2495764

[33] M Handel, L Mosher, Subgroup classification in $\operatorname{Out}\left(F_{n}\right)$ arXiv:0908.1255

[34] S Hersonsky, F Paulin, On the rigidity of discrete isometry groups of negatively curved spaces, Comment. Math. Helv. 72 (1997) 349-388 MR1476054

[35] I Kapovich, The frequency space of a free group, Internat. J. Algebra Comput. 15 (2005) 939-969 MR2197815

[36] I Kapovich, Currents on free groups, from: "Topological and asymptotic aspects of group theory", (R Grigorchuk, M Mihalik, M Sapir, Z Sunik, editors), Contemp. Math. 394, Amer. Math. Soc., Providence, RI (2006) 149-176 MR2216713

[37] I Kapovich, Clusters, currents, and Whitehead's algorithm, Experiment. Math. 16 (2007) 67-76 MR2312978

[38] I Kapovich, Random length-spectrum rigidity for free groups, Proc. Amer. Math. Soc. 140 (2012) 1549-1560 MR2869139

[39] I Kapovich, M Lustig, The actions of $\operatorname{Out}\left(F_{k}\right)$ on the boundary of outer space and on the space of currents: minimal sets and equivariant incompatibility, Ergodic Theory Dynam. Systems 27 (2007) 827-847 MR2322181

[40] I Kapovich, M Lustig, Geometric intersection number and analogues of the curve complex for free groups, Geom. Topol. 13 (2009) 1805-1833 MR2496058

[41] I Kapovich, M Lustig, Domains of proper discontinuity on the boundary of outer space, Illinois J. Math. 54 (2010) 89-108 MR2776986

[42] I Kapovich, M Lustig, Intersection form, laminations and currents on free groups, Geom. Funct. Anal. 19 (2010) 1426-1467 MR2585579

[43] I Kapovich, M Lustig, Stabilizers of $\mathbb{R}$-trees with free isometric actions of $F_{N}$, J. Group Theory 14 (2011) 673-694 MR2831965

[44] I Kapovich, T Nagnibeda, The Patterson-Sullivan embedding and minimal volume entropy for outer space, Geom. Funct. Anal. 17 (2007) 1201-1236 MR2373015 
[45] I Kapovich, T Nagnibeda, Geometric entropy of geodesic currents on free groups, from: "Dynamical numbers-interplay between dynamical systems and number theory", Contemp. Math. 532, Amer. Math. Soc., Providence, RI (2010) 149-175 MR2762139

[46] I Kapovich, T Nagnibeda, Generalized geodesic currents on free groups arXiv: 1105.5742

[47] I Kim, Ergodic theory and rigidity on the symmetric space of non-compact type, Ergodic Theory Dynam. Systems 21 (2001) 93-114 MR1826662

[48] I Kim, Marked length rigidity of rank one symmetric spaces and their product, Topology 40 (2001) 1295-1323 MR1867246

[49] I Kim, Rigidity on symmetric spaces, Topology 43 (2004) 393-405 MR2052969

[50] R Martin, Non-uniquely ergodic foliations of thin-type, measured currents and automorphisms of free groups, $\mathrm{PhD}$ thesis, University of California, Los Angeles (1995) MR2693216

[51] J-P Otal, Le spectre marqué des longueurs des surfaces à courbure négative, Ann. of Math. 131 (1990) 151-162 MR1038361

[52] F Paulin, The Gromov topology on $\mathbb{R}$-trees, Topology Appl. 32 (1989) 197-221 MR1007101

[53] B Ray, Non-rigidity of cyclic automorphic orbits in free groups, Int. J. Alg. Comp. 22 (2012) 1250021

[54] J Smillie, K Vogtmann, Length functions and outer space, Michigan Math. J. 39 (1992) 485-493 MR1182503

[55] K Vogtmann, What is . . outer space?, Notices Amer. Math. Soc. 55 (2008) 784-786 MR2436509

[56] B Zimmermann, Über Homöomorphismen n-dimensionaler Henkelkörper und endliche Erweiterungen von Schottky-Gruppen, Comment. Math. Helv. 56 (1981) 474-486 MR639363

\section{SST/IRMP}

Chemin du Cyclotron 2, bte L7.01.01, 1348 Louvain-la-Neuve, Belgium

Dipartimento di Matematica of the University of Bologna

Piazza di Porta S. Donato 5, 40126 Bologna, Italy

Department of Mathematics, University of Illinois at Urbana-Champaign

1409 West Green Street, Urbana IL 61801, USA

School of Mathematics, University of Southampton

Highfield, Southampton, SO17 1BJ, United Kingdom

mathieu.carette@uclouvain.be, francavi@dm.unibo.it,

kapovich@math.uiuc.edu, A.Martino@soton.ac.uk

Received: 3 June $2011 \quad$ Revised: 19 April 2012 